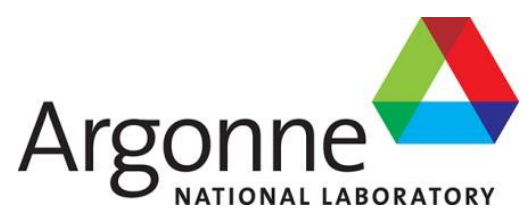

\title{
A Tool-Suite to Improve Reliability and Performance of Combined Transmission-Distribution Under High Solar Penetration
}

Energy Systems Division 


\title{
Acknowledgement
}

This material is based upon work supported by the U.S. Department of Energy's Office of Energy Efficiency and Renewable Energy (EERE) under the Solar Energy Technologies Office's Sunlamp Initiative, Award Number 31221.

\section{About Argonne National Laboratory}

Argonne is a U.S. Department of Energy laboratory managed by UChicago Argonne, LLC under contract DE-AC02-06CH11357. The Laboratory's main facility is outside Chicago, at 9700 South Cass Avenue, Argonne, Illinois 60439. For information about Argonne and its pioneering science and technology programs, see www.anl.gov.

\section{DOCUMENT AVAILABILITY}

Online Access: U.S. Department of Energy (DOE) reports produced after 1991 and a growing number of pre-1991 documents are available free at OSTI.GOV (http://www.osti.gov/), a service of the US Dept. of Energy's Office of Scientific and Technical Information.

\section{Reports not in digital format may be purchased by the public from the} National Technical Information Service (NTIS):

U.S. Department of Commerce

National Technical Information Service

5301 Shawnee Rd

Alexandria, VA 22312

www.ntis.gov

Phone: (800) 553-NTIS (6847) or (703) 605-6000

Fax: (703) 605-6900

Email:orders@ntis.gov

\author{
Reports not in digital format are available to DOE and DOE contractors from the \\ Office of Scientific and Technical Information (OSTI): \\ U.S. Department of Energy \\ Office of Scientific and Technical Information \\ P.O. Box 62 \\ Oak Ridge, TN 37831-0062 \\ www.osti.gov \\ Phone: (865) 576-8401 \\ Fax: (865) 576-5728 \\ Email: reports@osti.gov
}




\section{A Tool-Suite to Improve Reliability and Performance of Combined Transmission-Distribution Under High Solar Penetration}

prepared by

Karthikeyan Balasubramaniam

Energy Systems Division, Argonne National Laboratory

Date

08/26/2019 
Final Technical Report (FTR)

\begin{tabular}{|c|c|}
\hline a. Federal Agency & Department of Energy \\
\hline b. Award Number & DE-EE0001748 \\
\hline c. Project Title & $\begin{array}{l}\text { A Tool-Suite to Improve } \\
\text { Reliability and } \\
\text { Performance of Combined Transmission- } \\
\text { Distribution Under High Solar Penetration }\end{array}$ \\
\hline d. Principal Investigator & $\begin{array}{l}\text { Name: Karthikeyan Balasubramaniam } \\
\text { Title: Energy Systems Scientist } \\
\text { Email address: kbalasubramaniam@anl.gov } \\
\text { Phone number: } 630-252-9658\end{array}$ \\
\hline e. Business Contact & $\begin{array}{l}\text { Name: Judy Janovyak } \\
\text { Title: Financial Business Partner } \\
\text { Email address: jjanovyak@anl.gov } \\
\text { Phone number: 630-252-4988 }\end{array}$ \\
\hline f. Submission Date & 08/27/2019 \\
\hline g. DUNS Number & 624449968 \\
\hline h. Recipient Organization & Argonne National Laboratory \\
\hline i. Project Period & Start: 04/15/2016 \\
\hline
\end{tabular}

Acknowledgement: This material is based upon work supported by the U.S. Department of Energy's Office of Energy Efficiency and Renewable Energy (EERE) under SunShot National Laboratory Multiyear Partnership (SuNLaMP) initiative Award Number DEEE0001748. 


\section{Table of Contents}

1. Executive Summary

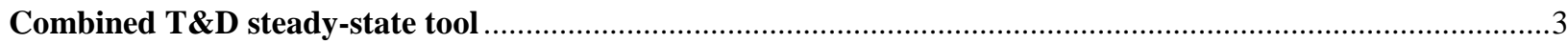

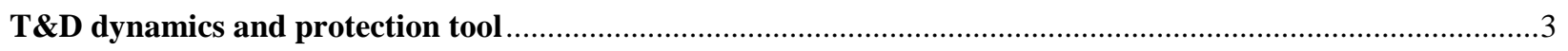

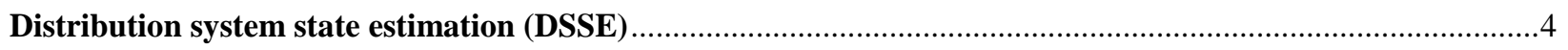

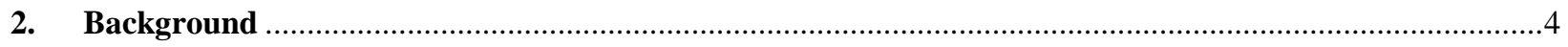

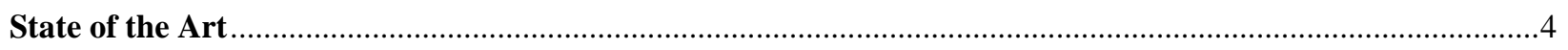

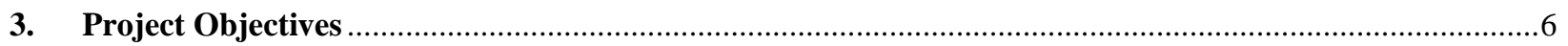

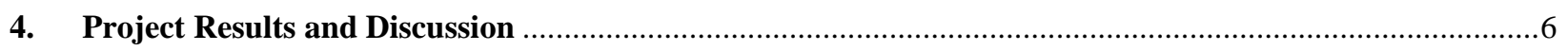

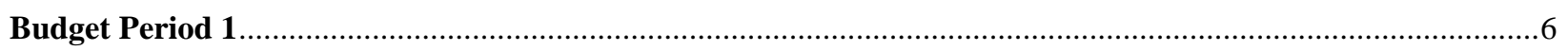

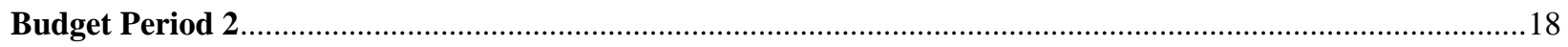

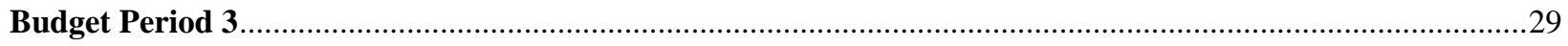

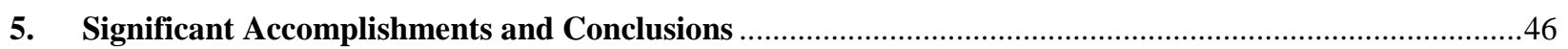

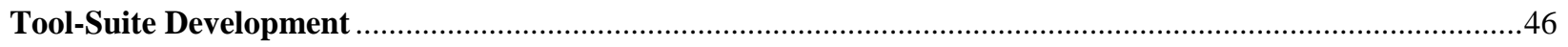

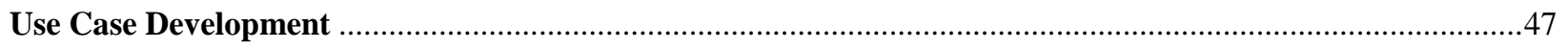

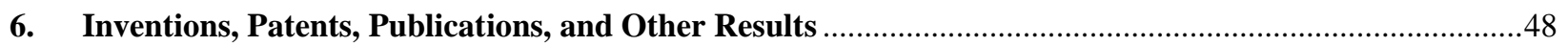

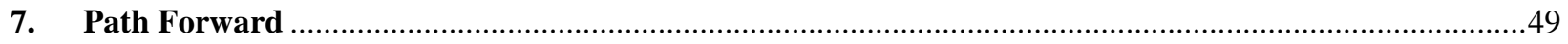

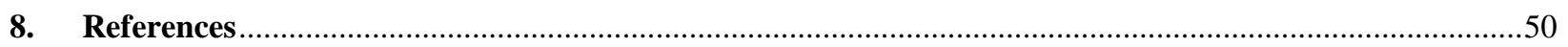


DE-EE0001748

Argonne National Laboratory

\section{Executive Summary}

The main motivating factor behind the work is to study the interaction between the transmission and the distribution system in more detail than currently possible. This is particularly important at high PV penetration levels. The developed toolkit allows capturing the coupling between the transmission and the distribution system (T\&D) for steady-state and dynamic analysis, thereby furthering our understanding of how the T\&D systems interact.

One of the objectives of the work is to answer the question, "which analysis require T\&D simulation". To this end, the team held discussions with the utility advisory group (UAG) and identified use cases that are of most interest and studied them in detail. Based on the use cases the team was able to suggest whether the T\&D analysis is a necessity for a particular study.

The developed techniques, which are documented in detail through publications, are important additions to the literature in power system co-simulation. The publications and the open sourced tools (PFLOW and IGMS) that resulted from this project are highly valuable resources to the researchers in this area. The team has also hosted a website, https://www.anl.gov/es/sunlamp-anl, to provide all the relevant information in a single place. Following is a high level summary of our findings using each of the developed tools,

\section{Combined T\&D steady-state tool}

The combined Transmission and Distribution simulation approach provided valuable information regarding the impacts of high penetrations of distributed solar generation on bulk power system operations. The detailed, individual advanced inverter models allowed different control strategies to be deployed and their impact on bulk power operating decisions to be studied. For example, changing the inverter control modes changed the optimal unit commitment and economic dispatch solutions. The ability for the system to maintain the balance between generation and consumption was also impacted based on the inverter control mode as was observed in the area control error. This granularity in asset modeling allowed for a deeper understanding of the relationship between transmission and distribution and how that might change in the future.

\section{T\&D dynamics and protection tool}

a) dynamics tool -- The UAG recommended a frequency response use case in which a transmission fault affects distributed solar PV inverters embedded in the distribution feeder. The objective of the study is to determine the degree to which an aggregated solar PV model, such as PVD1, captures the aggregated behavior of the PV inverters embedded in the distribution feeder. The concern is that high-penetration solar PV levels could lead to poor frequency response following a major transmission disturbance, which could initiate a cascading outage. Since the utility does not currently have the capability to study the combined T\&D system in detail, there is significant interest in validating an aggregated solar PV model, such as PVD1, under high-penetration scenarios. For the high-penetration scenario, the T-only model shows a similar post-fault oscillation as the combined T\&D model. However, this result cannot be generalized to other operating 
conditions, nor other penetration scenarios. In other words, there is no guarantee that a T-only model can capture the dynamics of a combined T\&D system for all operating conditions. In general, unbalanced operating conditions cannot be captured by traditional positive-sequence transient stability simulators. Therefore, combined T\&D models that assume positive-sequence balanced operation in the transmission system will fail to represent the true dynamics of a realistic power system under unbalanced conditions.

b) Protection tool -

- Any fault contingency in the transmission network is completely addressed and cleared by the transmission network protection and no coordination with the distribution network protection is necessary. Therefore, complete and correct analysis of transmission network protection of systems with high penetration of distributed generation based on rooftop photovoltaic panels is achievable exclusively by the analysis of transmission system models.

- Any fault contingency in the distribution network is completely addressed and cleared by the distribution network protection and no coordination with the transmission network protection is necessary. Therefore, complete and correct analysis of distribution network protection of systems with high penetration of distributed generation based on rooftop photovoltaic panels is achievable exclusively by the analysis of distribution system models.

\section{Distribution system state estimation (DSSE)}

The DSSE simulations were conducted with two different solution algorithms: SemiDefinite Programming (SDP) and Weighted Least Squares (WLS). The test was carried out on the modified IEEE 8500 node distribution test feeder with 2475 buses (3708 singlephase nodes). Three penetration scenarios, relative to peak feeder load, were simulated: $30 \%, 50 \%$, and $75 \%$. The feeder load for the testing snapshot was assumed to be $60 \%$ of the peak feeder load due to the timing of peak solar irradiance. The SDP chordal conversion implementation requires roughly 1.5 times more computation time than the WLS approach. Both SDP and WLS meet the $1 \%$ voltage magnitude error target.

\section{Background}

\section{State of the Art}

Historically, T\&D systems have been modeled separately, with very general assumptions made about the rest of the system. For distribution modeling, transmission is typically modeled as a perfect voltage source or Thevenin equivalent, while for transmission modeling the distribution system is treated as an aggregated load. However, with the introduction of large amounts of distributed solar photovoltaic (PV) power, the multi-scale interactions become critical, requiring rethinking these assumptions.

Others have performed some preliminary work using co-simulation for integrated transmission and distribution analysis. The GridSpice project [And2013] combines bulk power analysis using MATPOWER with distribution analysis using GridLab-D. However, 
this project apparently has not been in active development in more than two years. The FNCS project [Dai2014] has developed a promising co-simulation framework with links to GridLAB-D and MATPOWER. However, the system is still in early stages of development and its architecture does not appear to scale well to very large systems. NREL's IGMS takes a similar approach, yet provides a number of significant advantages:

- Ground-up support for highly scalable HPC applications,

- Automated tools for scenario generation, and

- A much more sophisticated simulation of transmission scale market operations and controls from day-ahead unit commitment down to 4-sec AGC using the NREL developed FESTIV model.

Dynamic timescale analysis has traditionally considered only the transmission scale with distribution feeders simplified to a single overhead line segment and aggregated representations of five different types of loads [Kos2010a, Kos2010b]. There has also been some effort to use parallel SPICE circuit simulation in HPC to model large-scale electric power system dynamics [Sch2011]. This pure electrical approach, however, does not adequately capture the electromechanical dynamics of large generators, and requires considerable conversion effort from existing power system dynamic models.

Recent efforts by Ellis et al. add a single aggregated PV system to the load bus and also introduce a simple, generic dynamic inverter model structure [Ell2011]. This was a major step forward, but fails to capture the important spatial variations for PV (and load) and the potential for heterogeneous inverter types and set points across a distribution feeder. In particular, during voltage disturbances, inverters connected at different points on a feeder can see very different voltage profiles, suggesting a need for either location-based voltage ride-through settings or wider ride-through envelopes for distribution connected systems than those used at the bulk power level. The proposed research will include a far more detailed representation of the distribution system, including multi-branched network representation to capture spatial differences and enable heterogeneity in the PV inverter fleet. The proposed work is novel in that it is believed to be the first integrated distribution and transmission system modeling effort utilizing HPC that can accurately describe the transmission level impacts of large amounts of distributed energy resources such as PV. The proposed simultaneous transmission-distribution (T-D) dynamics and protection simulation is novel. Traditional transmission dynamics simulators crudely coupled to distribution dynamics simulators will not produce faithful representations of dynamic grid behavior once distribution networks become more dynamic. Time-series analysis, power flow snapshots, and traditional fault studies fail to capture the necessary dynamics for identifying stability limits and protection limits for planning studies, especially under a high-penetration PV scenario. The team will continue its development of a new solution technique that solves the transmission-distribution system equations simultaneously (superior simultaneous "single equation" solution protocol) as opposed to merely connecting different transmission dynamics simulators with distribution dynamics simulators that exchange information at the end of every step (inferior serial or parallel interaction protocols).

For AC distribution systems, state estimation (SE) aims to minimize the weighted least squares problem that is typically nonlinear and non-convex. The Gauss-Newton (G-N) 
method is often applied to solve such a problem. However, the solution, if found, is always a local optimum. In some cases, the iteration process cannot even converge. Hence, a semidefinite programming (SDP) formulation will be developed in this work to solve the SE problem, which is relaxed to a solvable convex problem by the semidefinite relaxation (SDR). After the relaxation, the global optimum to the relaxed problem can be obtained. Then, rank reduction techniques provide a good starting point to find the global optimal solution of the original problem. A major benefit of the SDP method is its robustness, which will be helpful in the future of dynamic distribution operations with high penetration PV.

\section{Project Objectives}

Achieving very high penetrations of solar photovoltaic (PV) envisioned by SunShot will imply important changes to power system planning and operation. Of particular concern are the needs to improve understanding of transmission-distribution interactions, create an awareness of real-time distribution system operating conditions, and capture time series dynamics across multiple scales within associated tools. To overcome such technical barriers/issues, the project team will develop a suite of software tools that imparts a holistic understanding of the steady-state and transient behavior of transmission-distribution interaction under high PV penetration levels along with real-time monitoring of the distribution system and integration of system protection.

\section{Project Results and Discussion}

\section{Budget Period 1}

Reporting period: 04/15/16 - 04/14/17

\section{Task 1.1}

Setup and manage UAG

\section{Sub-task 1.1.1:}

Identify potential utilities with an emphasis towards utilities with high solar penetrations.

Summary of activities: The team targeted the following four primary, high-solar utilities as candidates for the UAG: (a) Southern California Edison, (b) Hawaiian Electric Company (HECO), (c) Arizona Public Service (APS), and (d) Pacific Gas and Electric (PG\&E).

\section{Sub-task 1.1.2:}

Establish connection with these utilities and invite them to be a member of the utility advisory group for knowledge and data sharing. 
Summary of activities: Invitation letters were sent to utilities. HECO, PG\&E, APS, and Southwest Power Pool (SPP) have provided verbal agreements or are considering participation in the project as utility advisors.

\section{Sub-task 1.1.3:}

Set up required contractual terms for data and information sharing.

\section{Summary of activities:}

- HECO has agreed to be the data-sharing partner for this project.

- A four-way NDA has been initiated to share the HECO data with the reset of the team.

- IIT will be investigating weak-grid scenarios through their dynamics simulation tool TS3ph.

- The data for typical relays found on the distribution system has been shared by HECO with Electrocon.

\section{Sub-task 1.1.4:}

Set up schedule for monthly meetings to provide and update on the project progress Summary of activities:

- The team decided to conduct its first utility advisory group meeting at the beginning of BP1 Q2.

- Monthly conference calls were conducted with HECO, PG\&E.

- The meeting minutes and conference call recordings were shared with the team.

\section{Sub-task 1.1.5:}

Share the comments from the UAG members with the team

\section{Summary of activities:}

- Discussed the following with the UAG: a) roadmap for the project, b) relay settings, c) special topics of interest for UAG members, $d$ ) validating $T+D$ simulator.

- Use case discussion on evaluation of voltage-VAR control - the overarching use case.

- Use case discussion on sensor selection for distribution system state estimation.

\section{$>$ T-1.1 Milestone:}

$\checkmark \quad$ Inclusion of minimum three (3) utilities within 3 months with one (1) agreeing to share data required for the use cases within six (6) months.

$\checkmark 9$ teleconferences and 1 webinar, and 1 annual meeting

\section{Summary:}

- T (1.1.a): The team formally completed the NDA execution with HECO for data sharing. A four-way NDA was executed between HECO, NREL, IIT, ANL, and Electrocon. The data received from HECO includes (a) distribution feeder data for four HECO feeders in Synergi format, (b) transmission steady-state data in PSSe format, 
(c) transmission dynamics data in PSSe format, (d) protection system data with locations and settings.

- T (1.1.b): Eight teleconferences have been conducted with UAG group since August 2016. The meeting minutes have been included in the quarterly reports.

This milestone is fully complete.

\section{Task 1.2:}

Develop use cases, scenarios, and benchmark comparison references for the developed tools.

\section{Sub-task 1.2.1:}

Gather information available in public domain on the use cases demonstrating the need for combined transmission-distribution analysis, with an emphasis on PV penetration.

\section{Summary of activities:}

Gathered use cases available in the public domain. This endeavor resulted in the following findings:

EPRI Smart Grid Repository [EPSR], ISGAN report on TSO-DSO interaction [ISGAN2014], [Hudson2012], [Margo2014], [Berdner2015].

\section{Sub-task 1.2.2:}

Establish use cases for each individual tool and an overarching use case for a combination of tools.

Summary of activities:

- The team developed a template for use cases.

- Gathered input from UAG members:

- Provide information to help engineers decide when three-phase modeling is necessary and when it is not

- Address concerns about voltage ride through.

- Address concerns about low short circuit ratios for weak grids where fault current is not much higher than load current.

- Address concerns about lack of visibility on the secondary side of the service transformers. Based on the above items, the team prepared a list of potential nine use cases that may be of interest to UAG. These use cases span all three tools being developed under the project.

- HECO and PG\&E have provided feedback to the team on the use cases. Based on the feedback, the team developed the following use cases:

- DER's impact on bulk system reliability: The study will analyze a combined T\&D test system with and without advanced distributed systems with high penetrations of distributed solar PV. 
- Voltage control by smart inverters: The main objective of this use case is to validate the effectiveness of VVC in maintaining steady-state feeder voltage profile within ANSI limits.

- DER's impact on frequency response: The main objective of this use case is to understand the ability of the electric power system to recover following disturbances under the stress of increasing renewable energy resources embedded in the transmission grid and increasing DER penetration in the distribution system. The study will analyze a test system using combined T\&D dynamics and protection simulation with high penetrations of distributed solar PV. Studying the impact on frequency response metrics can quantify this critical aspect of stability control under increasing levels of DER penetration.

\section{Subtask 1.2.3:}

Commence preparation of the simulation scenarios for the use cases

Summary of activities:

The team discussed various possible simulation scenarios that would supplement the use-cases. The potential scenarios are (a) weather conditions, (b) loading conditions, (c) different PV penetration levels, (d) different VAR control devices, (e) impact of disturbances (faults) at different locations, (f) different operational strategies. This list of potential simulation scenarios was discussed with the UAG at our last conference call. In addition to the above scenarios, the utility partners suggested to consider the following scenarios:

- Considering single as well multiple outages

- Inclusion and handling of cloud cover events

- Effect of unbalance as the DER penetration increases (due to increase in single-phase generation/load)

- PG\&E suggested to start with $10 \%$ PV penetration and going upwards of $100 \%$ feeder load. Similar numbers were suggested by HECO.

\section{Subtask 1.2.4:}

Begin gathering of data required for conducting simulations of the use cases and scenarios

\section{Summary of activities:}

The team is coordinating the data from the UAG partners to obtain the available data needed for the use cases and scenarios. HECO has agreed to provide the system data for conducting steady-state and dynamics simulations. PG\&E has twelve prototypical feeders available publicly that could be used.

\section{Subtask 1.2.5:}

Establish reference comparison and thresholds for validating the three simulation tools 
The team has selected the following potential reference comparison and thresholds for validating the three simulation tools. These references and thresholds were presented to the utility advisors at the last UAG meeting.

T\&D steady-state analysis tool: Voltage magnitude and real/reactive power comparison at the $T \& D$ boundary buses. The numerical threshold will be finalized in coordination with UAG. A $1 \%$ threshold for voltage magnitude and $5 \%$ for real/reactive load powers are reasonable estimates for consideration.

T\&D dynamics analysis tool: Largest voltage magnitude for steady-state solutions at three load buses and frequency comparison at minimum three generators. A $1 \%$ threshold for largest voltage magnitude and $1 \%$ for frequency will be used for validation.

Distribution system state estimator: A $1 \%$ threshold on voltage magnitude and angle would be used to validate the distribution system state estimator.

The UAG members suggested a $1-3 \%$ difference in voltage magnitude or angle as a threshold for validation and/or up to $5 \%$ difference in real and reactive power as an acceptable metric for validation.

\section{$>$ T-1.2 Milestone:}

Summary:

$\checkmark$ Define minimum one-use case for each individual tool and one overarching use case

$\checkmark$ Establish simulation scenarios for the use cases.

$\checkmark$ Establish benchmarking references for validating the three simulation tools

This milestone is fully complete.

\section{Task 1.3:}

Implement high-fidelity quasi-static combined transmission-distribution (T-D) analysis tool.

\section{Sub-task 1.3.1:}

Develop algorithm for coupling T\&D

\section{Summary of activities:}

The goal of this task is to integrate ANL's transmission system power flow solver PFLOW within NREL's Integrated Grid Modeling System (IGMS) tool using a loose-coupling scheme as shown in Fig. 1 and the loose-coupling protocol is illustrated in Fig. 2. 


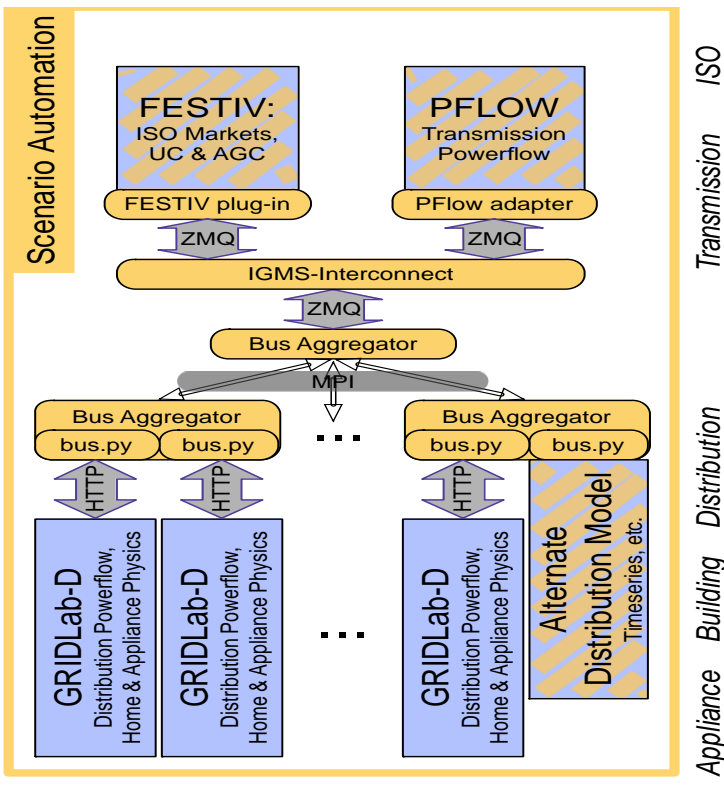

Figure 1. The Integrated Grid Modeling System (IGMS) with PFLOW.

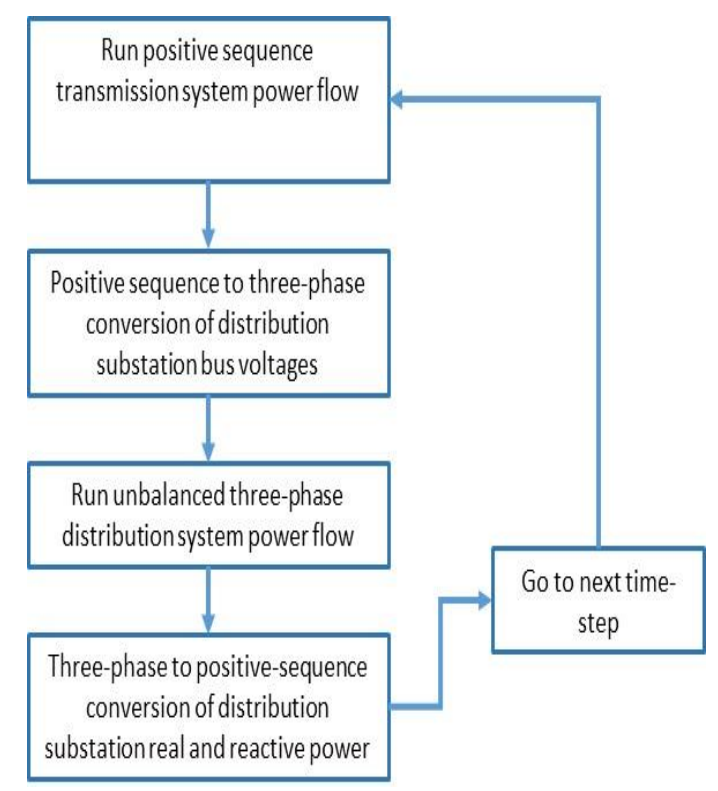

Figure 2. Flowchart for each time step of combined transmission-distribution steady-

- Implemented a loose-coupling scheme for the coupled T-D simulator that exchanges information between IGMS and PFLOW. In this approach, an iterative execution of unbalanced three-phase distribution system power flow followed by positive sequence transmission system power flow is performed at each step of the quasi-static analysis.

\section{Sub-task 1.3.2:}

Incorporate detailed subtransmission/distribution substation model

Summary of activities:

- The objective of this subtask was to better represent the seam between the transmission and distribution networks via detailed substation model. By incorporating the substation model, we can better capture the true nature of the seam between transmission and distribution system modeling.

- The substation is designed by connecting the transmission bus to a step-down transformer, which has a primary voltage of $23 \mathrm{kV}$ and a secondary voltage of $12.47 \mathrm{kV}$. A regulator is attached to the secondary of the transformer, with a band center of $12.99 \mathrm{kV}$.

- It was decided to perform initial analyses on the PJM 5-bus test system [PJM101].

Sub-task 1.3.3:

Obtain data from NREL and publicly available test cases 


\section{Summary of activities:}

The study team has decided to perform initial analyses on the PJM 5-bus test system. The system consists of 5 buses, 6 branches, 5 generators, and 3 loads. The topology of this system can be seen in Fig. 3. The detailed characteristics of this transmission system are publicly available and can be found in [PJM101].

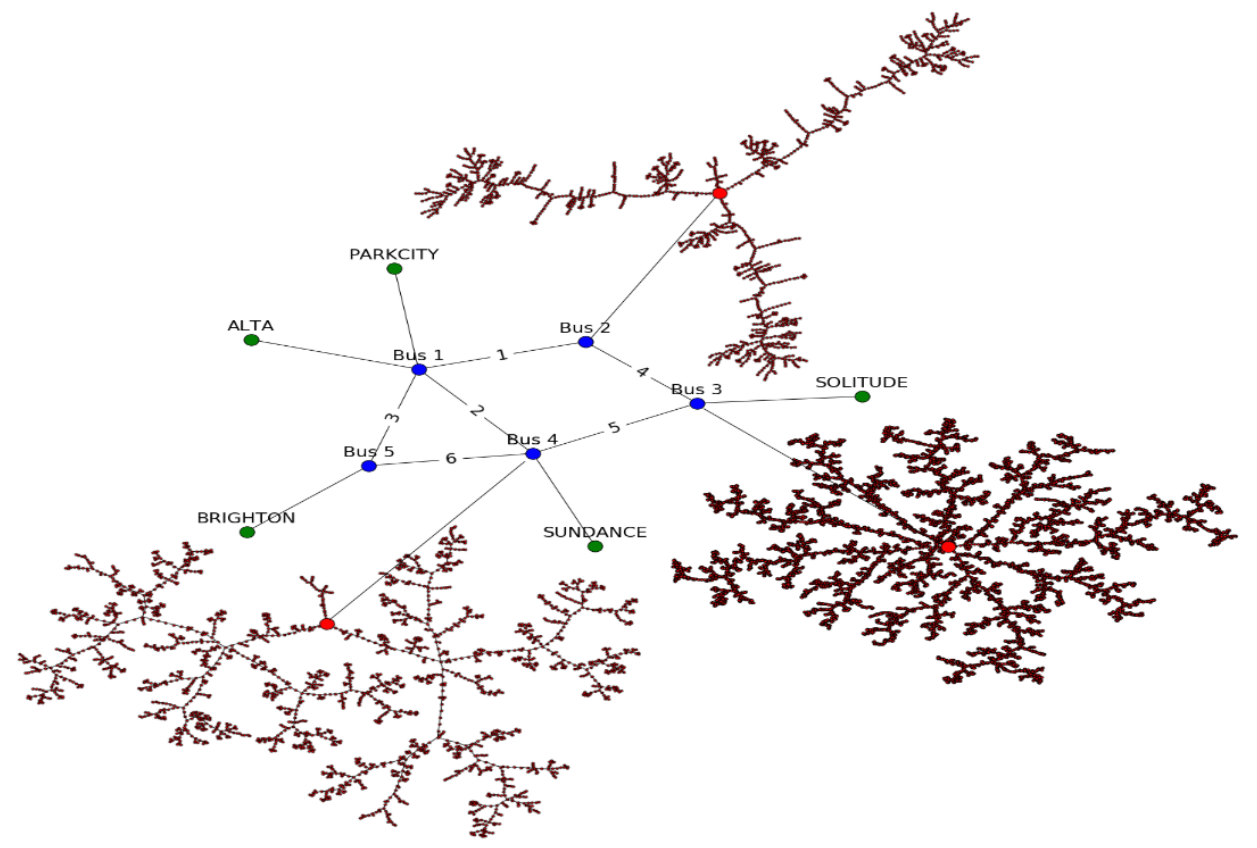

Figure 3. 5-bus, 11-feeder topology used for testing the functionality of the combined T\&D steady-state analysis tool.

\section{Sub-task 1.3.4:}

Interface ANL's transmission system simulator PFLOW with NREL's IGMS tool.

Summary of activities:

- Identified and implemented the interaction protocol (message passing) between IGMS and PFLOW. Message passing protocol: ANL's PFLOW is developed in C, while the FESTIV tool in NREL's IGMS is MATLAB-based. A MATLAB-based interface was first developed, followed by another message-passing protocol called ZeroMQ.

- Developed a wrapper in MATLAB and Python for PFLOW interface with IGMS.

- Evaluated four variants of IGMS-PFLOW interface.

- Selected the variant IGMS-PFLOW interface through ZMQ and Python due to its several advantages.

- Compared the performance of PFLOW against MATPOWER. For the IEEE-9 bus and 300 - bus test systems, PFLOW was $4 \mathrm{X}$ and 2.5X faster, respectively. 


\section{Sub-task 1.3.5:}

Conduct coupled T\&D simulations. Implement less than 5-second time-step for quasi-static analysis.

Summary of activities:

- Conducted case studies with the T+D analysis tool on PJM 5-bus test system extended with IEEE distribution test feeders and PNNL taxonomy feeders for three different distributed solar penetration scenarios.

- Made efforts to model the distribution feeder loads such that they reflected the behavior as seen in the field.

- Developed a software interconnect between ANL's transmission system simulator, PFLOW, and NREL's combined transmission and distribution simulation model, IGMS. PFLOW is connected to IGMS using a ZMQ interface. PFLOW is a parallel transmission system power flow that is built in C. IGMS integrates a market transmission system model via FESTIV in MATLAB with an operations-based quasisteady-state distribution system simulation using GridLAB-D.

- PFLOW can be used as a replacement for MATPOWER. IGMS sends generator real powers $(\mathrm{Pg})$, real power demands $(\mathrm{Pd})$ and reactive power demands $(\mathrm{Qd})$ to PFLOW and PFLOW return voltage magnitudes and angles. The interface is using ZMQ; the schematic for the data exchange is based on PFLOW's requirements. The data exchange is done in JSON.

- Prepared a report titled "Analyzing the steady-state impacts of high-penetration distributed solar on transmission system," which encompasses the different aspects of the T\&D steady-state simulation, including the tools used, coupling algorithm, data exchange protocols, and the impacts of increasing distributed solar on transmission system.

\section{$>$ T-1.3 Milestone:}

Summary:

$\checkmark$ Successful benchmarking on 6 out of 8 simulation scenarios (Each simulation scenario consists of 1 transmission network with 3 or more distribution networks with $1 \mathrm{PV}$ penetration level scenario)

$\checkmark \quad$ Less than $1 \%$ for voltage magnitude and less than $5 \%$ for lumped load OR other metric decided on subtask 1.2.5 (a)

\section{$\checkmark$ This milestone is fully complete.}

\section{Task 1.4:}

Implement simultaneous transmission-distribution dynamic stability and protection analysis tool

\section{Subtask 1.4.1:}


Add ability to read CYME distribution ASCII files that contain equipment, network, and other data into TS3ph

\section{Summary of activities:}

IIT implemented the ability to read CYME distribution ASCII input files (e.g., equipment, network, load) into TS3ph (merge from dNetSim). The CYME data files from an old version of CYME (version 5 from 2009) can be read correctly. The IEEE 13 Node Test Feeder system has been loaded into TS3ph and the computed solution matches the results presented in the IEEE 13 Node Test Feeder document. This test confirms the correct modeling of line and cable equipment presented in the IEEE 13 Node Test Feeder document.

\section{Subtask 1.4.2, 1.4.3, 1.4.4 and 1.4.5:}

Summary of activities:

- Successfully implemented and tested the dNetSim code (ported from MATLAB to C) in TS3ph for the IEEE 13 node test feeder.

- Successfully tested and verified the steady-state initialization process in TS3ph for the following test cases: IEEE 13 Node Feeder System, IEEE 34 Node Feeder System, and the IEEE 123 Node Feeder System. Verification was completed with both the IEEE Distribution Subcommittee Analysis reports and OpenDSS. Note that the IEEE documents and OpenDSS show slightly different results, but the differences are minor, i.e., on the order of $10^{\wedge}(-4)$ in per unit. The TS3ph steady-state initialization values agree with the same order of precision.

- For the combined transmission and distribution modeling effort in TS3ph, the team has successfully implemented a substation example based on a prototypical crossover design shown below. Note that this initial test did not include the $1 / 2$ ohm inductor, so the neutrals were directly grounded. The transmission model was based on the so-called WECC 9-bus system (a looped transmission network). The substation model was connected to bus 5 , which is a load bus in the 9-bus system, via two parallel lines. The original bus 5 load was split in a 9:1 ratio between the two $12.5 \mathrm{kV}$ buses. OpenDSS was used to verify the TS3ph results. The largest mismatches across the entire system was as follows:

\begin{tabular}{|l|l|l|l|}
\hline & Phase A & Phase B & Phase C \\
\hline $\begin{array}{l}\text { Maximum } \\
\text { Magnitude } \\
\text { Mismatch (p.u.) }\end{array}$ & 0.00021 & 0.00021 & 0.00022 \\
\hline $\begin{array}{l}\text { Maximum Angle } \\
\text { Mismatch (degrees) }\end{array}$ & 0.04 & 0.04 & 0.04 \\
\hline
\end{tabular}




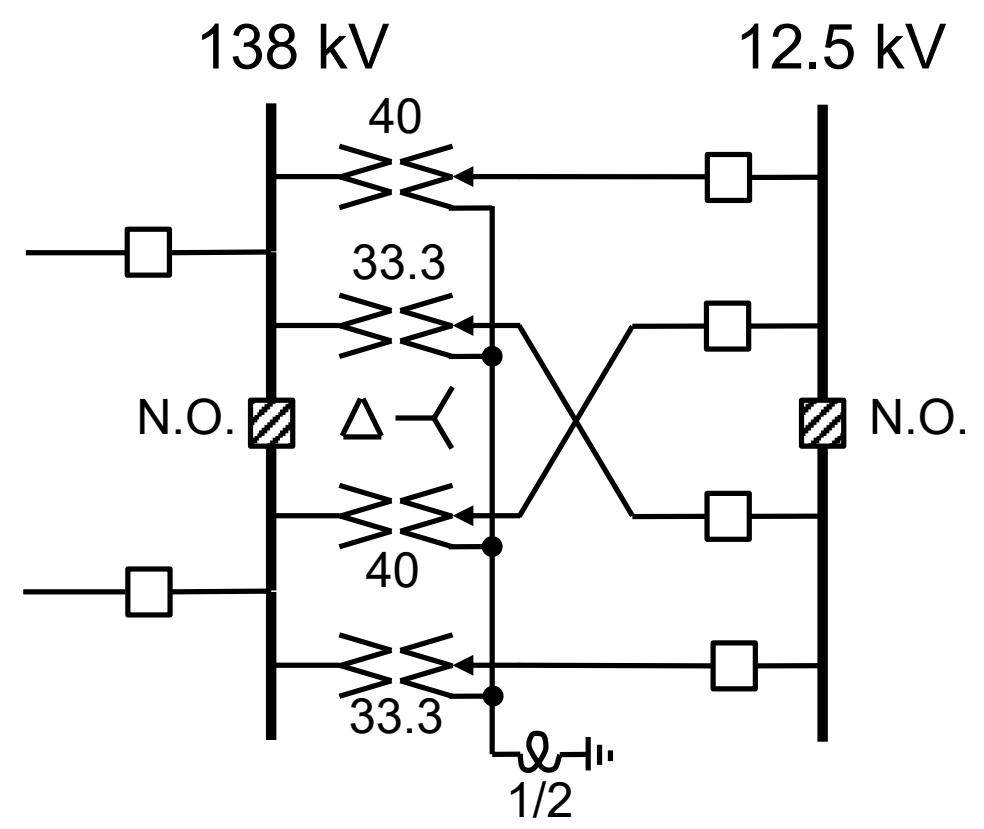

Figure 4. Prototypical substation configuration used in validation

\section{Subtask 1.4.6:}

Add PV phasor-based inverter model to TS3ph (best practice model from ANL and NREL) Summary of activities:

The PV phasor-based inverter model implementation has begun with an investigation of the Type 4 wind turbine model used in PSLF and PSSE, as documented in the WECC Solar PV report. Additional input from ANL and NREL will be incorporated in Q4.

\section{Subtask 1.4.7:}

Add single-phase induction motor, with rotor-stall feature for air-conditioning load to TS3ph (merge from IIT MATLAB dynamics simulator)

\section{Summary of activities:}

The single-phase induction motor with rotor-stall feature for air-conditioning load has been implemented in TS3ph. The performance of the single-phase induction motor following a normally-cleared fault matches the performance that was established in the IIT MATLAB dynamics simulator. The present work is focused on implementing the rotor-stall feature.

\section{Subtask 1.4.8:}

Implement distribution system relays in CAPE (based on the available utility data and recommendation of the UAG members) and integrate with TS3ph substation model

\section{Summary of activities:}

TS3ph simulation results following a single-line-to-ground fault have been verified against CAPE-TSLink, which uses PSSE for the positive sequence dynamics simulation. Based 
on the unbalanced fault testing, TS3ph is ready to pass voltages and currents to CAPE for evaluation of distribution system relays.

Details or general instruction for setting typical protective relays are required from HECO and PG\&E. Additionally, information of ratios of CTs and VTs by voltage level are necessary. Instances of protective relays will be deployed in the distribution system model according to HECO and PG\&E instructions. Once instances have been deployed, connected to their model CTs and VTs and proper setting have been provided, testing will be performed in CAPE to assure correct operation of protective relay models under typical CAPE studies.

\section{$>$ T-1.4 Milestone:}

$\checkmark$ Successful benchmarking on 6 out of 8 simulation scenarios (Each simulation scenario consists of 1 transmission network with 3 or more distribution networks with $1 \mathrm{PV}$ penetration level scenario)

$\checkmark \quad$ Less than $1 \%$ for voltage magnitude for steady-state solutions OR other metric decided on subtask 1.2 .5 (b)

\section{Benchmarking results: WSCC 9-bus with multiple copies of IEEE 13-node test feeders}
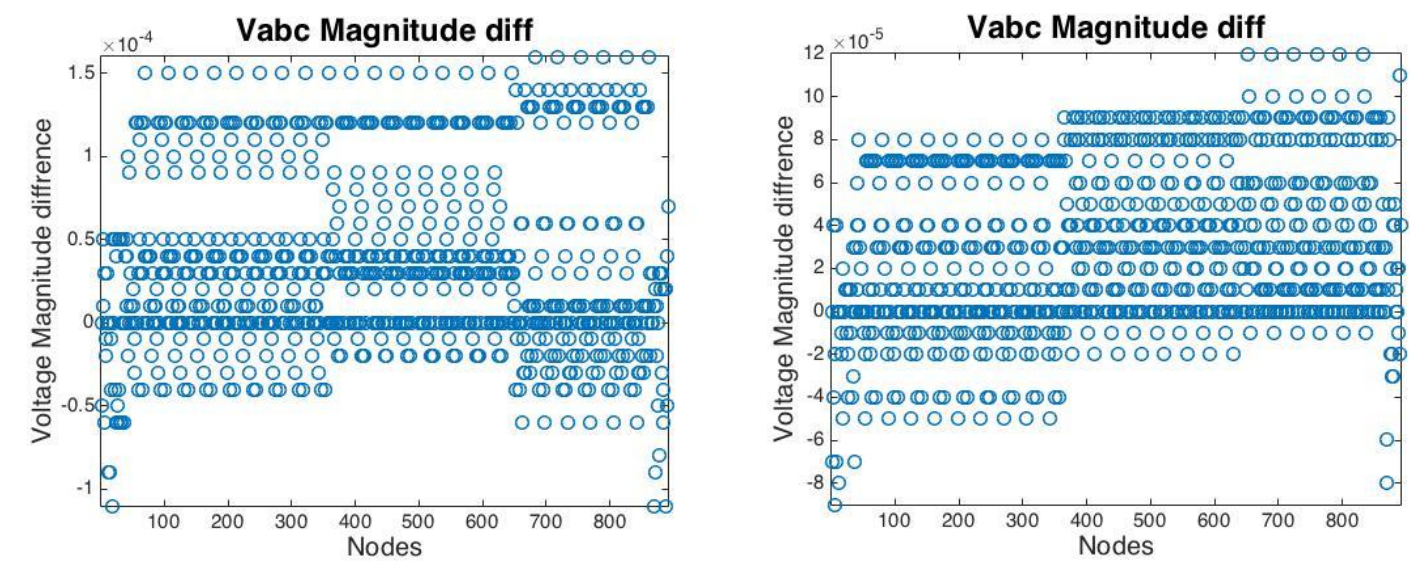

Figure 5. Voltage magnitude error (pu). Left: $30 \%$ penetration. Right: $60 \%$ penetration. All errors less than $10^{-2}$ in magnitude

\section{This milestone is fully complete.}

\section{Task 1.5:}

Distribution system state estimator development.

\section{○ Sub-task 1.5.1:}

Extend balanced 'per-phase' DSSE SCADA-based algorithm to include three-phase unbalanced distribution network.

Summary of activities: 
- Explored using the DSSE application to provide input data to some downstream applications.

- Formulated DSSE based on semidefinite programming formulation to handle unbalanced three-phase networks.

\section{○ Sub-task 1.5.2:}

Design semidefinite programming algorithm for three-phase unbalanced distribution network.

\section{Summary of activities:}

- Implemented the semidefinite programming algorithm for three-phase unbalanced distribution system state estimation.

- The state estimation problem assumes various measurements are available from the field, although many of the measurements do not directly measure the distribution system state.

\section{○ Sub-task 1.5.3:}

Benchmark SDP approach.

\section{Summary of activities:}

- To benchmark the SDP-based approach for DSSE, the team has compared the known power flow solutions of the IEEE test feeder systems to the estimates generated by the proposed SDP algorithm and a traditional weighted least squares approach for DSSE.

- During the benchmark tests, it became clear that the 2-norm of the state estimation errors for the IEEE 13-Node Test Feeder will become worse as the noise magnitude increases.

\section{Benchmarking results: IEEE 13-node test feeder}

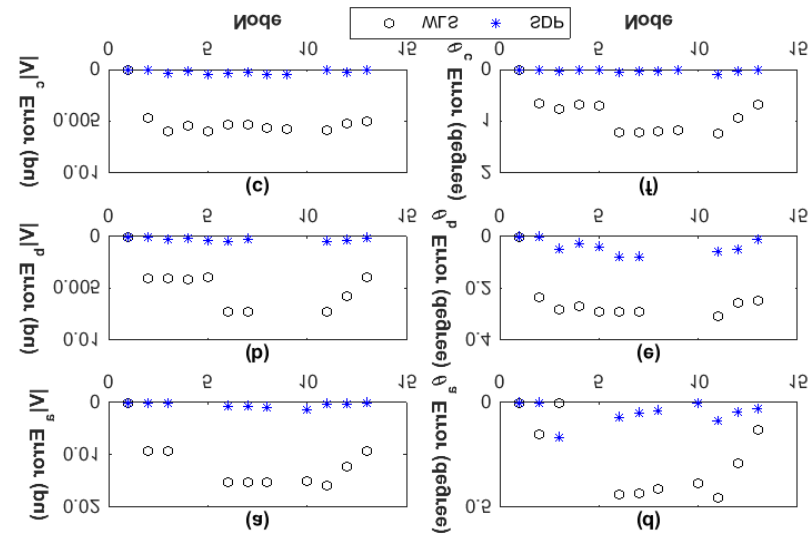

Figure 6. Voltage magnitude error (pu), voltage angle error (deg), all SDP errors less than $1 \%$ 


\section{T-1.5 Milestone:}

At least 7 out of 9 simulation scenarios should meet the success value of less than $1 \%$ error in voltage magnitudes and angles or metric based on subtask 1.2 .5 (c)

This milestone is fully complete.

Year-end summary - Go/no-go + milestone status:

- Include three utilities in advisory group and demonstrate functionality of the three tools.

$\checkmark$ HECO, PG\&E, and SPP are members of the Utility Advisory Group.

$\checkmark$ Achieve preliminary functionality of the three tools.

$\checkmark$ Completed Phase 1 milestones by end of phase except for

$\mathrm{X}$ Validation with CYME.

\section{Budget Period 2}

Reporting period: 04/15/17 - 04/14/18

\section{Task 2.1:}

Continue developing the combined transmission-distribution steady-state analysis tool to address UAG scenarios, and utilization of real-utility data.

\section{Sub-task 2.1.1:}

Integrated utility transmission and distribution data into a form for comprehensive T\&D analysis with IGMS.

Summary of activities: The team converted raw T\&D dataset from HECO to .xml format. Then the DiTTo tool was used to convert from OpenDSS to GridLAB-D. Validation was done by running power flow and comparing voltage profiles.

\section{Sub-task 2.1.2:}

Develop an efficient prototype Windows-based wrapper for IGMS.

Summary of activities: An interface called Bus.py was developed to pass messages between one or more GridLAB-D instances and IGMS using a ZeroMQ socket. This ZeroMQ Bus was used to develop a OpenDSS Remote Server based on OpenDSSDirect.py to interact with OpenDSS.

\section{Sub-task 2.1.3:}

Prototype at least one additional analytics. 


\section{Summary of activities:}

- A new analytic was proposed to go beyond the power factor boundary condition and better understand the stability margins appropriate to enforce on distribution in a high DER future.

- Traditionally, the (voltage) stability margins for a transmission load bus are computed using continuation power flow to trace bus voltages throughout the system and to identify the critical loading level.

- It is possible to trace out much of the stable region of these nose curves using traditional NR power flow.

- This realization makes it possible to use NR power flow to estimate the $Q$ stability margin while keeping $P$ fixed. Specifically, it is possible to trace out the noncontingency $\mathrm{P}-\mathrm{Q}$ stability envelope using the following steps:

- Identify transmission level nodes of high importance/value from T-only analysis.

- Select a desired (net) power demand for the load bus of interest and fix this value.

- Varying the corresponding $Q$ value at ever increasing demand levels. For each, run a power flow solution to check for stability

- The $Q$ demand level at which the NR power flow no longer converges, is then taken as a proxy for the critical point and retained as the $\max Q$ demand at that $P$ demand level.

- Repeat steps 2-4 for different P demand levels. Optionally re-dispatching the system generation for each new $\mathrm{P}$ demand level.

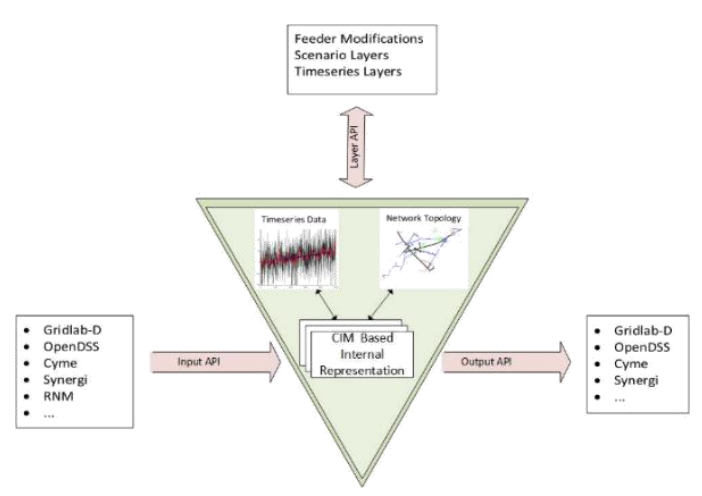

Figure 7. DiTTo workflow.

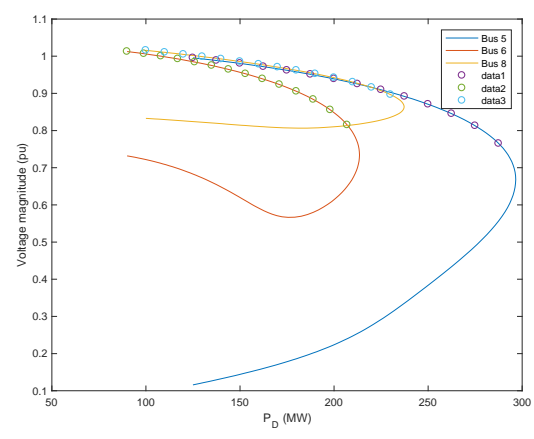

Figure 8. Traditional Voltage Stability Margins represented using "nose" curves.

\section{Sub-task 2.1.4:}

Test and refine model of real world T\&D data needed for scenarios identified in 1.2.3. 


\section{Summary of activities:}

The integrated T\&D model based on the UAG data was finalized and five scenarios were created based on the level of distributed solar PV generation simulated.

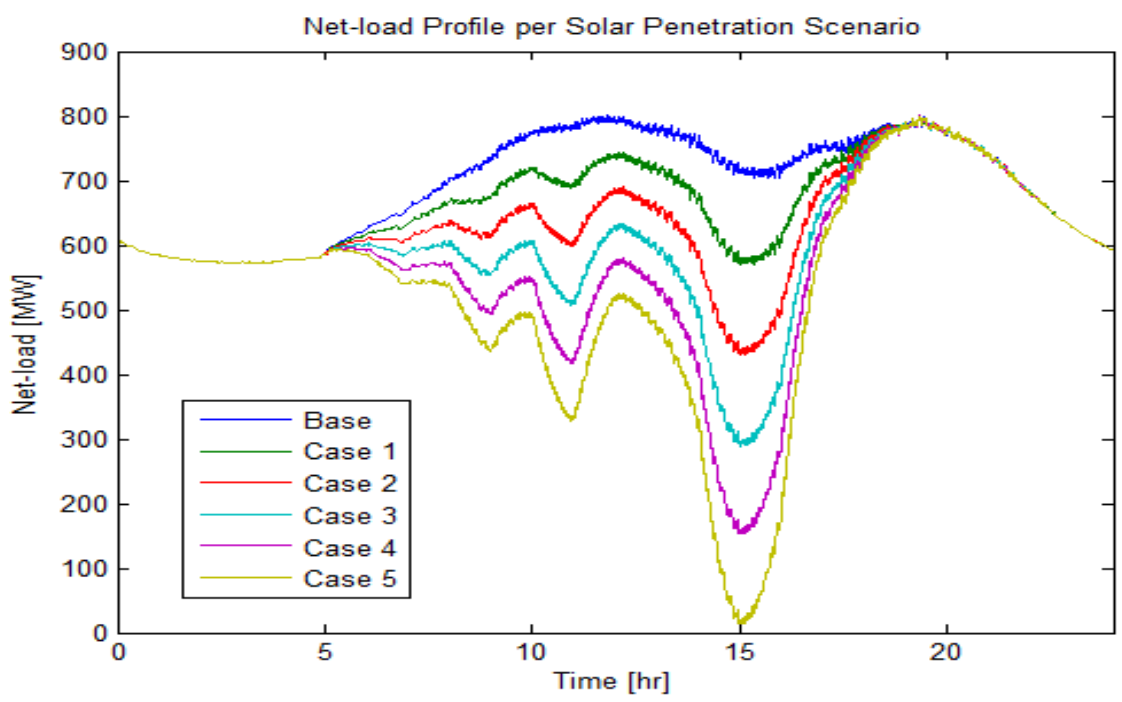

Figure 9. Net load profile for each solar penetration scenario considered

\section{Sub-task 2.1.5:}

Distribution system model validation, for simulations of BP1.

\section{Summary of activities:}

The voltages of the 13 and 123 node distribution feeders were calculated using GridLAB$D$ and compared with the IEEE provided voltage values. The maximum absolute error in the voltage magnitudes across all the three phases was 0.0027 p.u., while the maximum absolute error in the voltage angles was 0.12 degree for 13 node feeder. The maximum absolute error voltage magnitudes across all the three phases was 0.0018 p.u., while the maximum absolute error in the voltage angles was 0.21 degree for 123 node feeder.

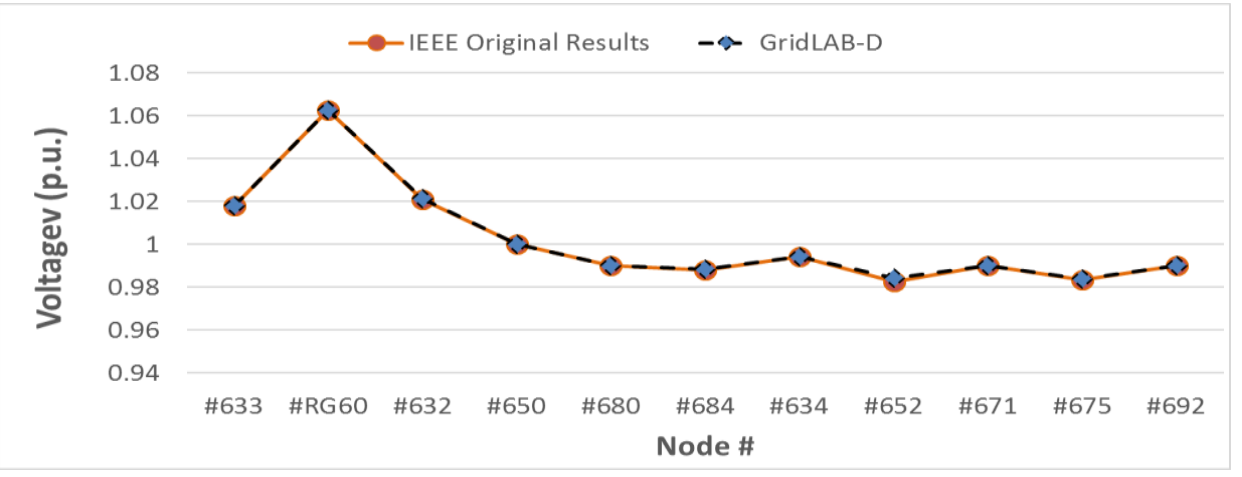

Figure 10. Phase A Voltage Phasor Magnitudes (Max. Absolute Error: 0.0017 p.u.) 


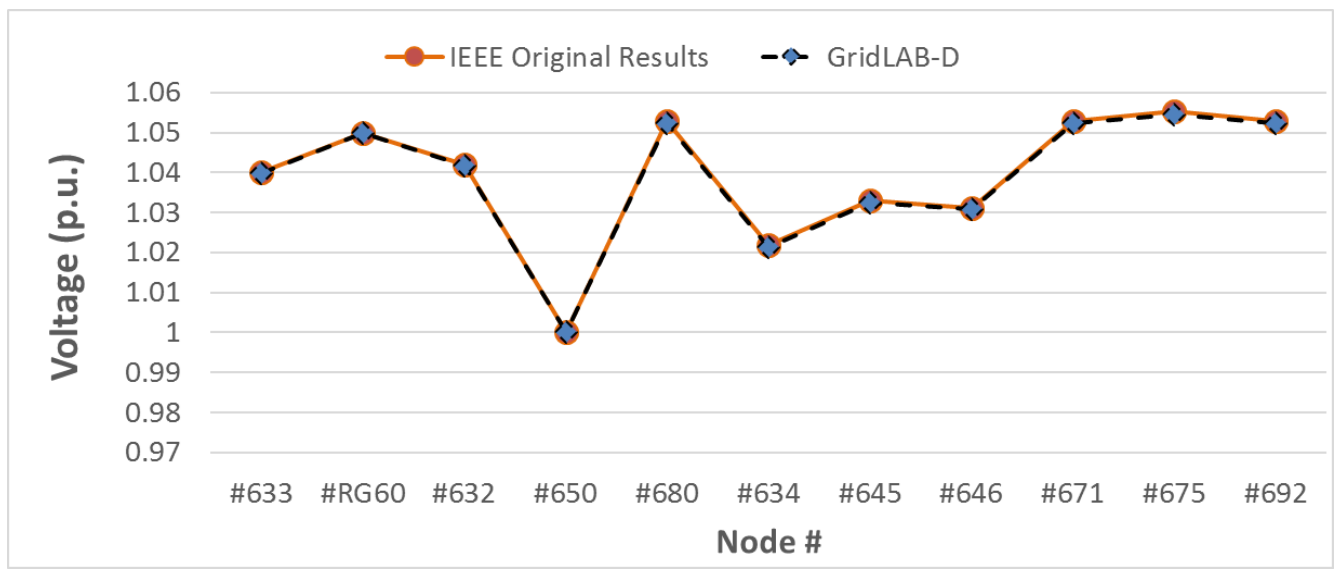

Figure 11. Phase B Voltage Phasor Magnitudes (Max. Absolute Error: 0.0006 p.u.)

\section{Subtask 2.1.6:}

Establish one new use-case and one expanded GMLC use-case.

\section{Summary of activities:}

- A use case based on the GMLC use-case 'Adaptive Volt-VAR control at high PV penetration' was developed to define steady-state voltage stability boundary limits as a function of the active and reactive power injection limits on select transmission buses. The impact of advanced PV inverter Volt-Var/power factor controls schemes on these stability limits will be studied and quantified.

- Another use-case on assessing the impacts of system protection on combined transmission-distribution dynamics was proposed.

\section{$>$ T-2.1 Milestone:}

Completed data preparation and Windows modeling software implementation in IGMS.

- The data preparation as per subtask 2.1.1 was completed on Oct 14, 2017. The implementation for the Windows tool in IGMS as per subtask 2.1.2 was completed on Jan 14, 2018.

Complete transmission for service area with full sub-transmission plus distribution for at least 2 substations and at least 7 feeders able to run without fatal errors.

- The successful simulation of the integrated T\&D system per subtask 2.1.4 was successfully completed on April 15, 2018.

- Minimum one (1) additional use-case and one expanded GMLC use-case with varying $P V$ penetration scenarios

- The additional use case per subtask 2.1.6 was completed on April 15, 2018.

\section{$\checkmark$ This milestone is fully complete.}




\section{Task 2.2:}

Implement dynamic phasor models of PV inverters.

\section{Sub-task 2.2.1:}

Identify gaps in existing dynamic phasor models for PV inverters.

Summary of activities:

A survey of existing work on modeling of PV inverters found that EMT models can model intricate details but are computationally intensive while approximate phasor models lacks the smart inverter functionality or model the impact of change in irradiance.

\section{Sub-task 2.2.2:}

Develop prototype of improved dynamic phasor solar generator model.

Summary of activities:

- The equations for PV inverter controllers, current limiting, and PV panel characteristics were developed.

- Features like phase locked loop, Volt-VAR and Volt-WATT smart inverter functions, voltage ride through as per IEEE 1547, and PV array dynamics were implemented

- Detailed specifications for each were described in the model specifications document.

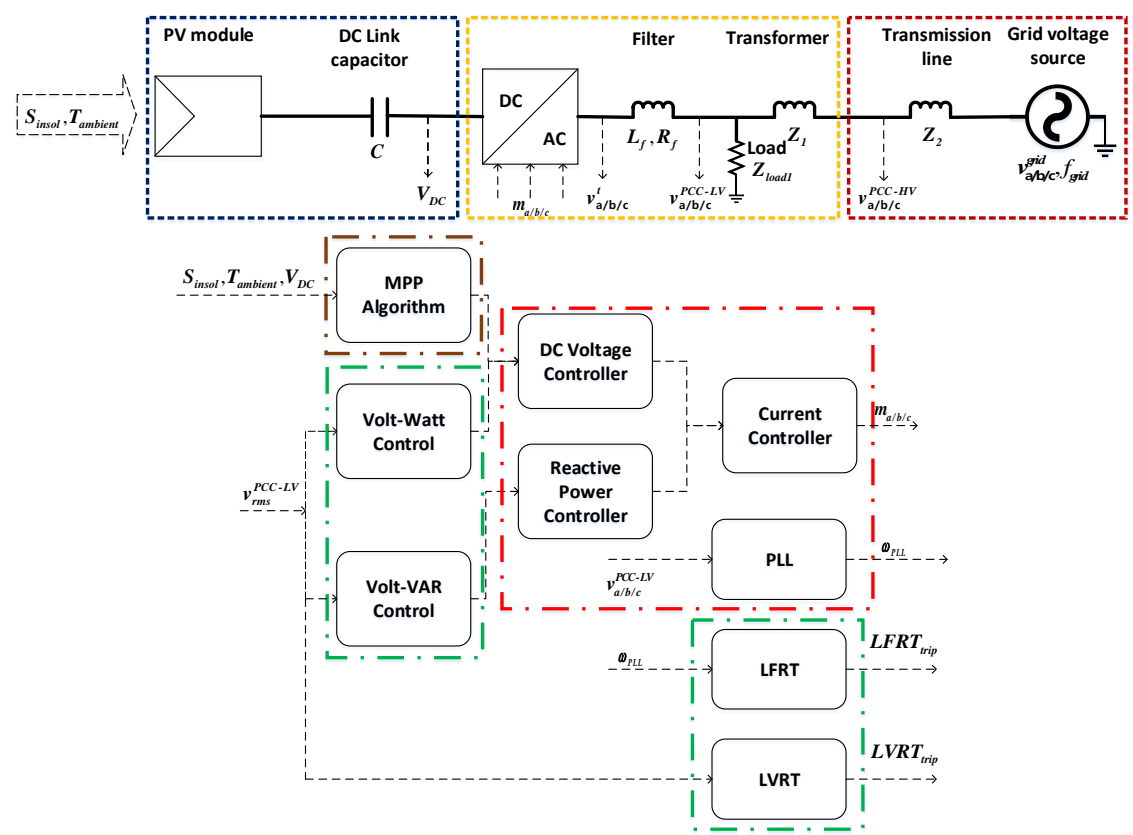

Figure 12. Grid-connected solar PV-DER model. 


\section{Sub-task 2.2.3:}

Coordinate with IIT on the implementation of the dynamic phasor inverter model in the combined transmission-distribution dynamics simulation.

Summary of activities:

The source code, block diagrams, and the simulation results were provided to IIT for implementation of the model in TS3ph for evaluation.

\section{Sub-task 2.2.4:}

Run model for validation/benchmarking.

Summary of activities:

The new model was benchmarked against PSCAD and SimScape for 5 simulation scenarios.

\section{$>$ T-2.2 Milestone:}

$\checkmark$ Completed prototype implementation of dynamic phasor models and its benchmarking on minimum five different scenarios.
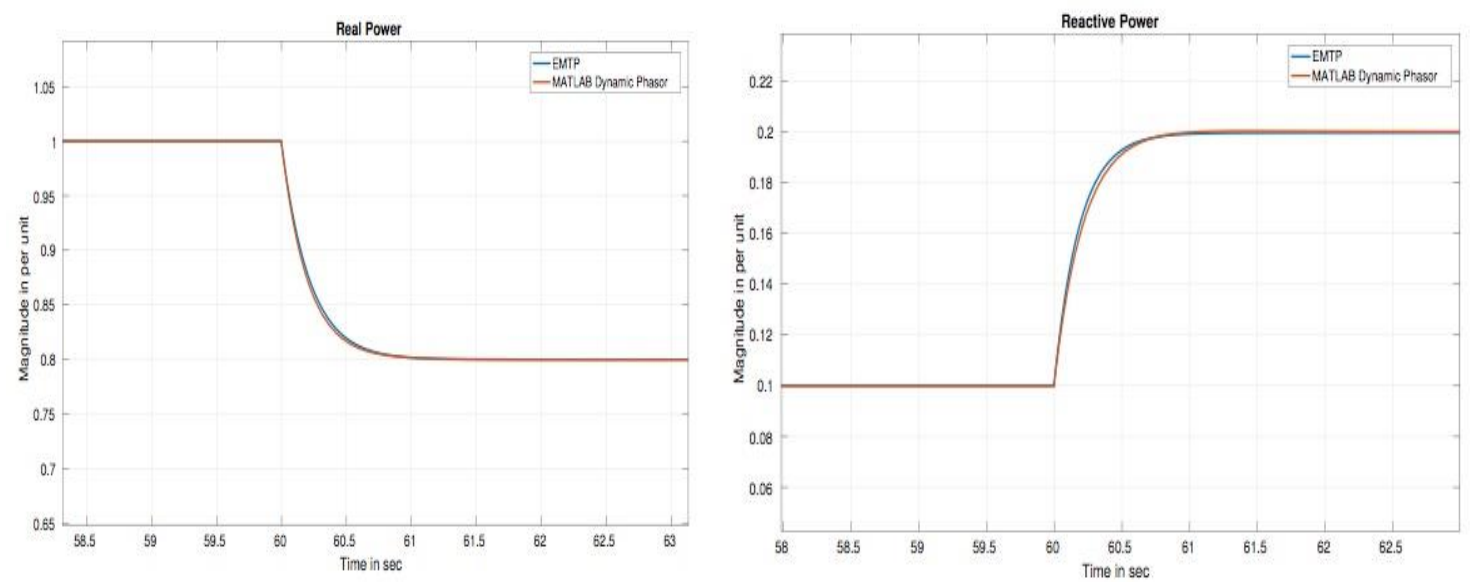

Figure 13. Model validation of dynamic phasor model. Left: real power control. Right: reactive power control.

\section{$\checkmark$ This milestone is fully complete.}

\section{Task 2.3:}

Development of simultaneous Transmission-Distribution dynamic stability and protection analysis was continued.

\section{Sub-task 2.3.1a:}

Add and evaluate electromagnetic transient (EMT) interface to TS3ph for detailed source modeling (merge from IIT MATLAB TSEMT simulator). 


\section{Summary of activities:}

- A review of the literature revealed several papers on hybrid simulation where phasorbased positive-sequence transient stability (TS) tools coupled in some way to electromagnetic transient (EMT) tools.

- Investigated use of EMT tools SimPowerSystems and PSCAD for verifying the future results obtained by TS3ph including fault conditions.

- It was found that either an EMT interface or a phasor interface can be used to connect the traditional generators and PV inverters.

- It was decided to implement an EMT interface and an EMT model of a PV inverter will be incorporated into TS3ph.

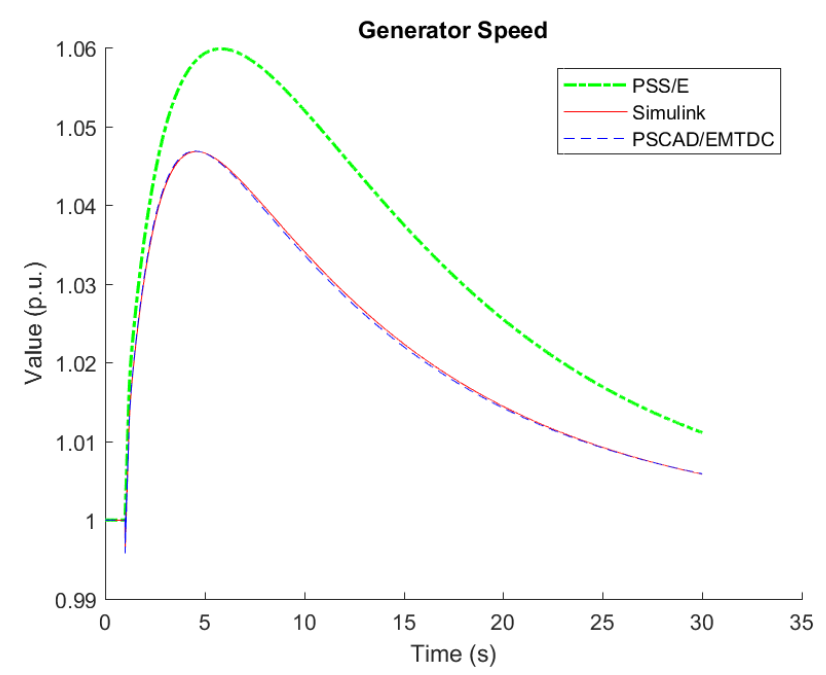

Figure 14. Different between PSSE and EMT simulators.

\section{Sub-task 2.3.1b:}

Add PV phasor-based inverter model to TS3ph.

Summary of activities:

- IIT implemented a set of PV phasor models based on the PVGU1 series in PSS/E.

\section{Sub-task 2.3.1c:}

Implement combined T\&D protection in CAPE for HECO case.

\section{Summary of activities:}

- An NDA was signed with HECO permitting Electrocon to receive PSS/E data and details of relay elements required for protection studies.

- A detailed substation model has been created as a proxy for the actual substation topology. 
- Preparation of the CAPE model for a portion of the HECO system was started by Electrocon.

\section{Sub-task 2.3.2:}

Add and evaluate electromagnetic transient (EMT) interface to TS3ph for detailed source modeling (merge from IIT MATLAB TSEMT simulator).

\section{Summary of activities:}

IIT has studied various approaches to model generators in TS3ph for combined T\&D dynamics simulations as well as evaluated a phasor based (similar to those in PSSE and PSLF) and two EMT based detailed generator models.

\section{Sub-task 2.3.3:}

Compare PV EMT-based inverter model to best practice from ANL and NREL.

\section{Summary of activities:}

- A report explaining the development and testing of one EMT reference model and another phasor-based model were delivered to IIT.

- A simplified average PV model was developed by IIT, which replaced the power electronic switches and the LCL filter with a controlled voltage source and a series inductor. When compared with the detailed switching model it was found to accurately capture the PV inverter dynamics during and after the fault and had computational time savings of $17 \%$ and $38 \%$.
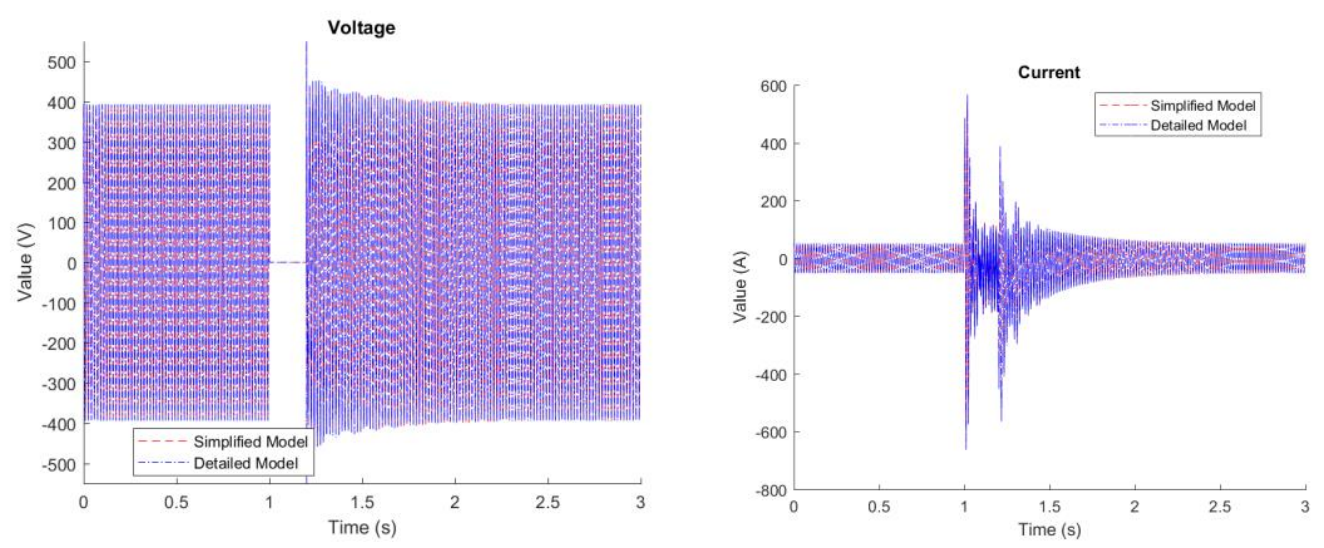

Figure 15. Fault simulation comparison.

\section{Sub-task 2.3.4:}

Implement distribution relays in CAPE and test TS3ph-CAPE with five (5) test cases.

Summary of activities:

- Discussions on the distribution relays and associated protection schemes necessary to prepare for high PV penetration levels were conducted with the UAG. 
- A three-phase unbalanced four-bus subsystem from the IEEE 13 Node Test Feeder was used to benchmark the simplified average PV inverter model against a single-phase detailed switching PV inverter model,

- The simplified model matches the detailed model with less than $1 \%$ error in voltage magnitude after a period of two cycles following each switching event.

T-2.3 Milestone:

At least 4 out of 5 transient scenarios simulated should meet the success rate of less than $1 \%$ error, or success metric based on subtask 1.2 .5 (b), for the largest average magnitude percentage differences between voltage states.
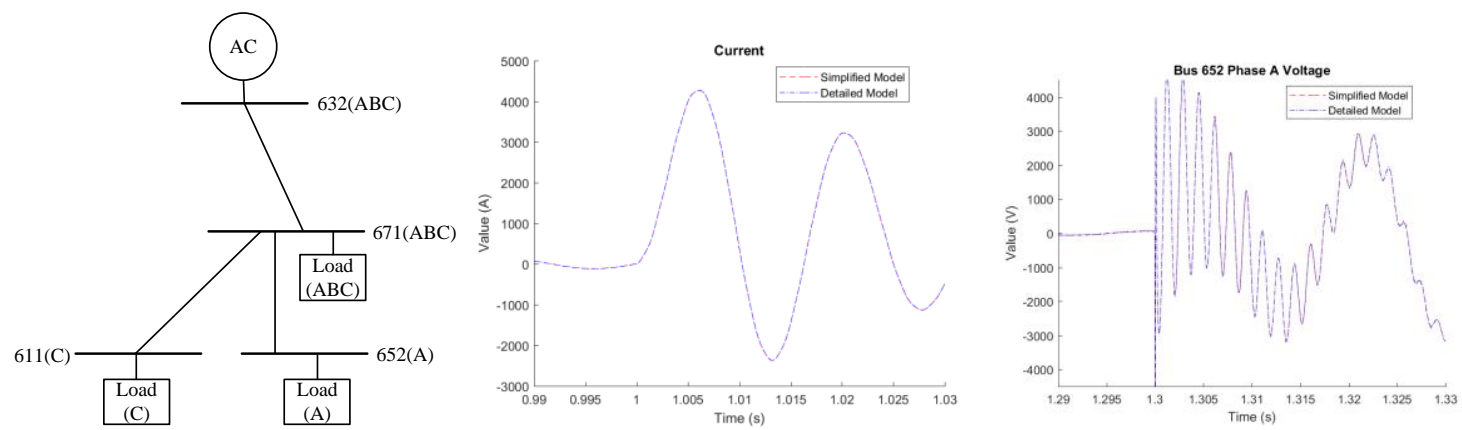

Figure 16. Left: Single-phase PV model connected to subsystem of IEEE-13. Middle: Fault-on at 1.0s: No current harmonics. Right: Fault-off at $1.3 \mathrm{~s}$ : Voltage harmonics appear. (|Vsimple| $\mid$ Vdetailed $\mid) / \mid$ Vdetailed $\mid=0.53 \%$ two cycles following switching event

\section{$\checkmark$ This milestone is fully complete.}

Task 2.4:

The development of the distribution system state estimator was continued.

\section{Sub-task 2.4.1:}

Add framework for new data sources (e.g. AMI, DA, and PMU).

\section{Summary of activities:}

- Based on the UAG members' input, a modification to the semi-definite program (SDP) approach was initiated to include current magnitude input from recent current sensors with noise levels ranging from 2 to $10 \%$.

- Detailed comparisons between SDP and weighted least squares (WLS) with a set of 50 to 100 noisy measurements have yielded a probability density plot of state estimation errors. It was found that SDP has smaller errors compared to WLS but can take up to 5 times longer for 13 and 34 node feeder systems.

- A new data framework to include measurements from smart meters, distribution automation equipment etc. and an optional "noise generator. 


\section{Sub-task 2.4.2:}

Incorporate AMI (smart meter) data measurements.

Summary of activities:

Since smart meter data was not available from utility partners, synthetic data was created by adding Gaussian noise to a power flow solution.

\section{Sub-task 2.4.3:}

Incorporate DA event data measurements.

Summary of activities:

- Since distribution automation data was not available from our utility partners, synthetic data was created by adding Gaussian noise to a power flow solution.

- The extended SDP formulation with current magnitude sensor data was tested with following procedure:

- Solve the three-phase unbalanced power flow

- Identify the locations and measurement types for each sensor

- PMU: voltage magnitude and angle, current magnitude and angle

- Current magnitude sensor: current magnitude

- Multiply the true power flow based values (voltages and currents) by a noise factor sampled from a Gaussian distribution with mean 1.0 and standard deviation as described below:

- PMU: voltage and current magnitude and angle noise standard deviation $=0.002$

- Current sensor: current magnitude noise standard deviation $=0.1$.

- Estimate the complex voltages based on the noisy measurements.

- Calculate the errors between the voltage estimates and the original true values from the power flow solution.

\section{Sub-task 2.4.4:}

Incorporate PMU event data measurements.

\section{Summary of activities:}

Synchrophasor data was not available from our utility partners, so synthetic data was created by adding Gaussian noise to a power flow solution. Micro PMU synchrophasor data measurements were included in the SDP formulation, as well as substation PMU data. The results in subtask 2.4.3 illustrate the ability of the SDP algorithm to achieve the $1 \%$ error target, even when AMI data is not available.

\section{Sub-task 2.4.5:}

Simulation for voltage error evaluation. 


\section{Summary of activities:}

- The DSSE solution was compared with the original power flow solution and the SDP and WLS estimation errors were computed.

- To provide evidence for Milestone 2.4, a series of DSSE problems were solved using the IEEE 123 Node Test Feeder with noisy data, missing data and bad data.

- To produce a robust evaluation of the DSSE approach by sampling from the Gaussian noise factor distribution, 100 scenarios were run for each set of DSSE problems.

\section{$>$ T-2.4 Milestone:}

Successful benchmarking on at least 4 out of 6 simulation scenarios with less than $1 \%$ error or success metric based on subtask 1.2.5.c on the largest magnitude percentage voltage error.

Summary: Based on the SDP DSSE simulation results in the previous section, the voltage magnitude errors were less than $1 \%$ for more than $67 \%$ of the simulations, so Milestone 2.4 has been achieved.

Note: SDP computation takes $1.75 x$ time as that of WLS.
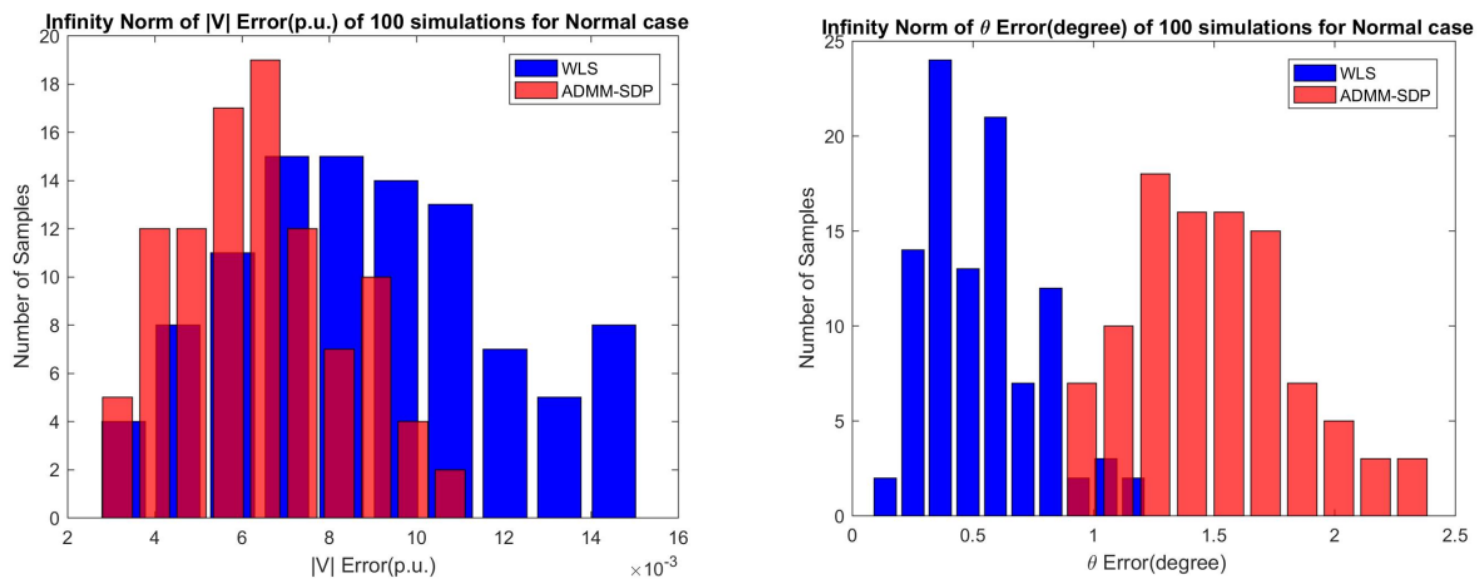

Figure 17. IEEE 123 Node Test Feeder. Substation PMU and AMI smart meters. Voltage measurement noise $=2 \%$. Current measurement noise $=10 \%$. Left: voltage magnitude error. Right: voltage angle error. $2 / 100$ had $|\mathrm{V}|$ error $>1 \%$ for ADMM-SDP.

This milestone is fully complete.

Year end summary - Go/no-go + Milestone status:

$\checkmark$ The Go/No-Go milestone for this second phase of the project was to ensure the milestones for tasks 2.1, 2.2, 2.3, and 2.4 were achieved.

$\checkmark$ The results presented for each of the simulator development task demonstrate the extended functionality of each tool.

$\checkmark$ The phase milestones have been achieved by end of BP2 Q4. 


\section{Budget Period 3}

Reporting period: 04/16/18 - 07/14/19

\section{Task 3.1:}

Use-case simulation and analysis with quasi-static combined transmission-distribution analysis tool

\section{Sub-task 3.1.1:}

Continue preparation of use-cases and PV penetration scenarios in conjunction with UAG members

\section{Summary of activities:}

The team continued to prepare the use case based on the feedback from UAG

\section{Sub-task 3.1.2:}

Conduct simulations and analysis of the use-cases.

\section{Summary of activities:}

Steady-State Impact of DERs on Bulk Power Operations:

- The steady-state impact of DERs on bulk power systems was completed and results will be presented during the review presentation.

- Final combined T\&D system defined in BP2 utilized and the following scenarios considered after discussions with the UAG:

- Base, Low (40\%), Medium (60\%), and High PV (80\%) penetration scenarios

- For the high PV scenario, the following sensitivities around inverter control mode were studied:

- Traditional unity power factor control schemes

- Constant 0.95 power factor control schemes

- Volt-Var control schemes based on Hawaii Rule 14.

- For each of these control schemes, varying levels of netload forecast error was considered:

- $50 \%$ netload forecast error

- $25 \%$ netload forecast error

- $10 \%$ netload forecast error

- $0 \%$ netload forecast error

- Analysis shows that: 
- As expected, increased levels of distributed PV increases net load variability and uncertainty, negatively impacting ACE metrics while providing reducing the overall system production cost

- High levels of distributed PV tend to bias the ACE of the system slightly positive (more tendency to overgenerate electricity)

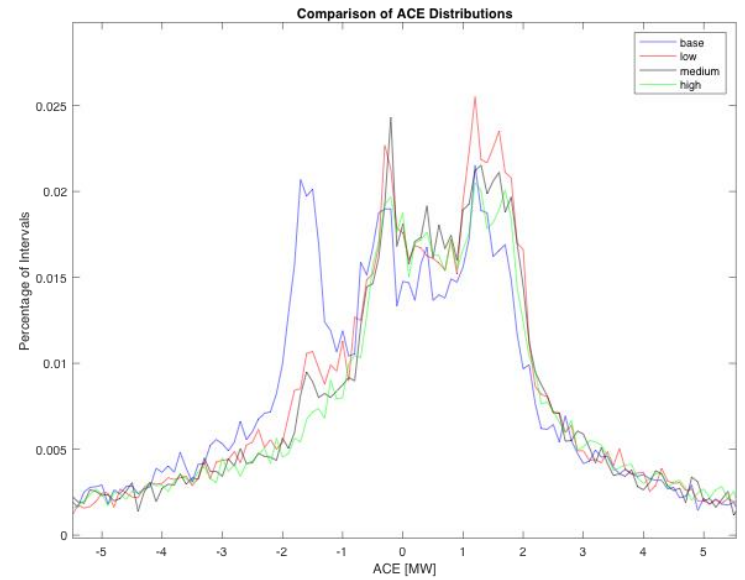

Figure 18. Comparison of ACE distributions

- Inverter control mode impacts system voltages as seen by the bulk power system (aggressive Volt-Var control schemes can help depress voltages)

- Inverter control mode impacted the rate at which ACE is accumulated in the system, particularly during the evening ramp (serves as limited ramping capacity and can ease the ramping burden on thermal generators)

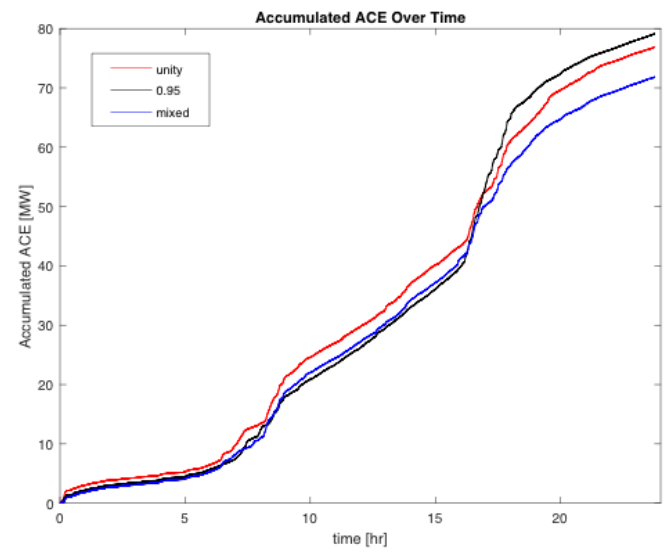

Figure 19. Accumulated ACE over time

- Inverter control mode impacted the commitment decisions made by the system operator 
- Inverter control mode impacted the amount of dispatch infeasibilities. The VoltVar control scheme helped reduce this the most since they also help mitigate the slope of the evening ramp.

o These results were presented to the UAG on July 25, 2019.

- Feedback from PG\&E and HECO was overall positive.

- Both expressed interest in any follow-on work that would potentially include protection (to study potential reverse power flow due to increase DPV) and advanced inverter controls for DPV.

\section{Beyond Power Factor:}

- Now using MATLAB Continuation Power flow

- $>40 \%$ faster $25 \mathrm{sec} \rightarrow 14 \mathrm{sec}$
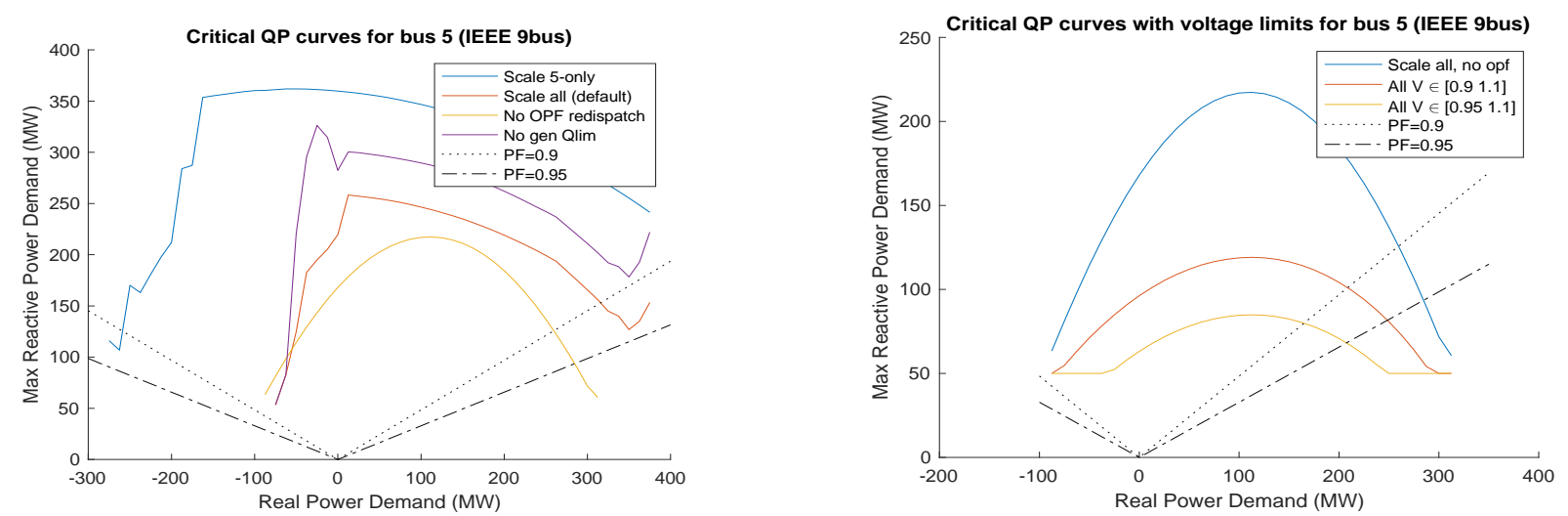

Figure 20. Critical QP curve with and without voltage limits at bus 5 on IEEE 9-bus test system

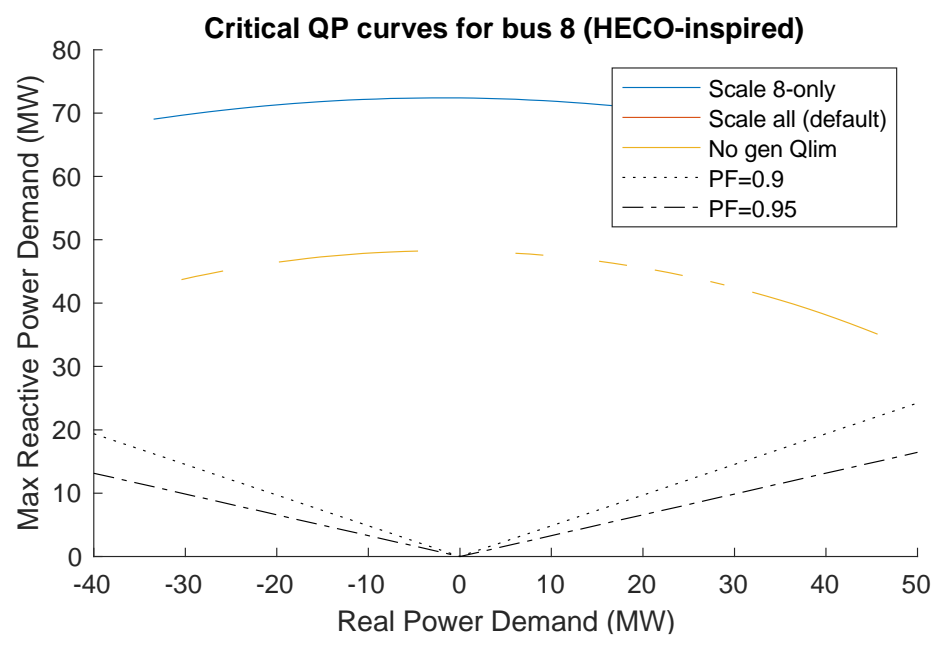

Figure 21. Critical QP curve at bus 8 of HECO-inspired test system 
- Common HECO-inspired test system

- Extracted from IGMS simulation run

- Status: limited convergence range

Please note that the team performed detailed analysis using standard IEEE test case but only produced initial results using HECO-inspired system - the test system that was used in all use cases.

\section{Sub-task 3.1.3:}

Document the findings in the final report

Summary of activities:

The findings from the use case have been documented

\section{Sub-task 3.1.4:}

Validate the tool against commercial tools

Summary of activities:

\section{Methodology:}

The following approach was used,

1. Run the quasi-static combined T\&D tool with the given load shape and solar shape. Save the interface voltage magnitude results.

2. Run the quasi-static D-only simulation tool (GridLAB-D) with the given load shape and solar shape. Set the corresponding voltage obtained from step 1 at the substation. Generate results and save the real and reactive power load as seen at the substation for each time step.

3. Run the quasi-static T-only simulation tool (MATPOWER) using the real and reactive power load obtained from step 2. Generate the results and save the voltage information for T-D interface.

4. Compare the voltage profile obtained in step 1 and 3 . The results of this step is shown in Fig. 22 (left) - voltage comparison and Fig. 22 (right) - voltage difference histogram below.

\section{Objective:}

The comparison results from step 4 should be within the required tolerance of $1 \%$ voltage magnitude error as asserted by the UAG. 

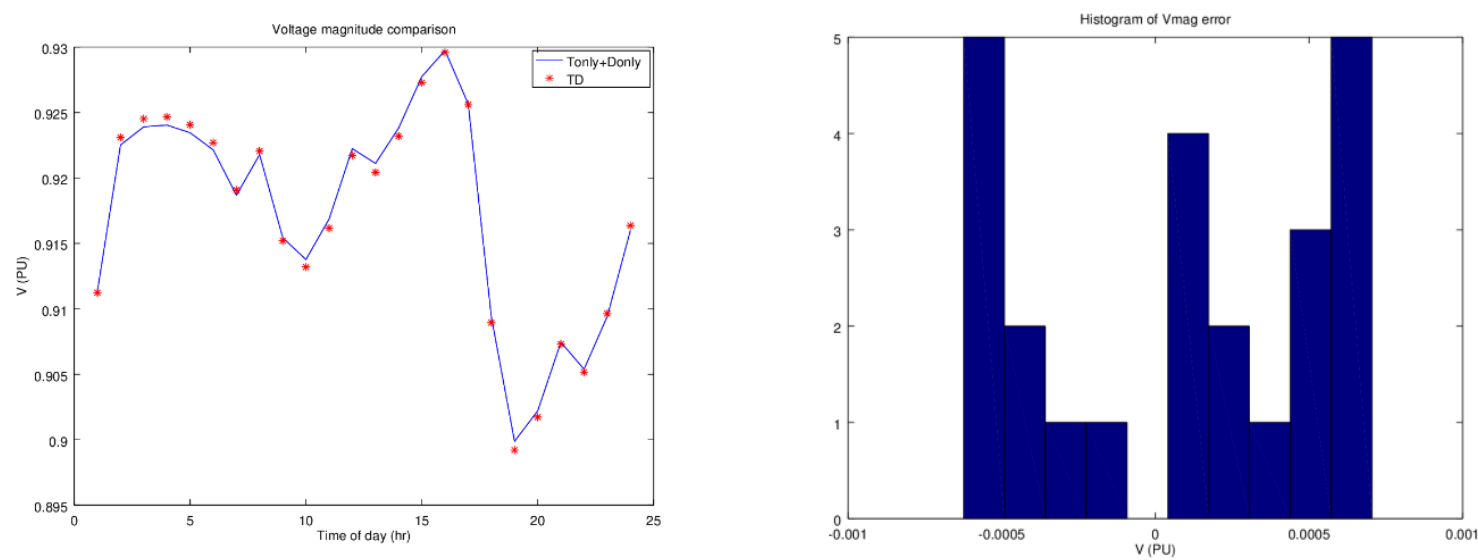

Figure 22. Left: Voltage comparison: Comparison of combined T\&D solution versus the solution methodology described above. This plot represents an independent assessment of the T\&D steady-static co-simulation tool. It verifies whether the tool works as it is supposed to. It serves as quantitative validation of milestone 3.1. Please note that during each dispatch the amount of solar generation relative to feeder peak load varies i.e. both the load shape and solar shape profile was used. Right: Voltage difference histogram: Histogram of error distribution for voltage magnitude difference at T\&D interface. The difference is within 0.1 percent.

\section{Milestone 3.1:}

$\checkmark$ At least 2 out of 3 PV penetration level simulation scenarios should meet the success value of less than $1 \%$ error on voltage magnitude and less than $5 \%$ error on lumped or metric based on subtask 1.2.5.a

$\checkmark$ This milestone is fully complete.

\section{Task 3.2:}

Analyze utility use-case with simultaneous transmission-distribution dynamic stability and protection analysis tool.

\section{Sub-task 3.2.1:}

Verify steady-state behavior of new TS3ph distribution system dynamic models against CYME

\section{Summary of activities:}

- The T\&D dynamics model with a balanced three-phase transmission network and an unbalanced three-phase distribution network were loaded into TS3ph and initialized for transient stability simulation. The initial operating point, including all complex voltages, was compared to a power flow solution from OpenDSS and found to match with less than $1 \%$ error.

- Performed comparison between TS3ph and OpenDSS for a post-contingency operating point such as the tripping of a line. 


\section{Sub-task 3.2.2:}

Verify dynamic behavior of new TS3ph dynamic models against PSSE

Summary of activities:

- Several tests were done with various faults and two different types of PV inverter models to verify the dynamics behavior of TS3ph, with PowerWorld Simulator as benchmark.

- The PVD1 model in PowerWorld was used which has real and reactive power inputs as well as Volt-VAR controller and protection elements for voltage and frequency violations.

- A more flexible PV inverter model for use in unbalanced distribution systems known as PVD1D was implemented. TS3ph can use the PVD1D model in a three-phase or single-phase configuration.

\section{Sub-task 3.2.3:}

Implement enhanced dynamic phasor solar generator model in the combined transmission-distribution simulation.

\section{Summary of activities:}

- A Python version of the dynamic PV phasor model has been written and given to IIT. Also, the dynamic phasor model's performance for different disturbances was evaluated.

- Implement enhanced dynamic phasor solar generator model in the combined transmission-distribution simulation.

- Response of the PV-DER model was improved by tuning the control loop parameters and adding anti-windup algorithms.

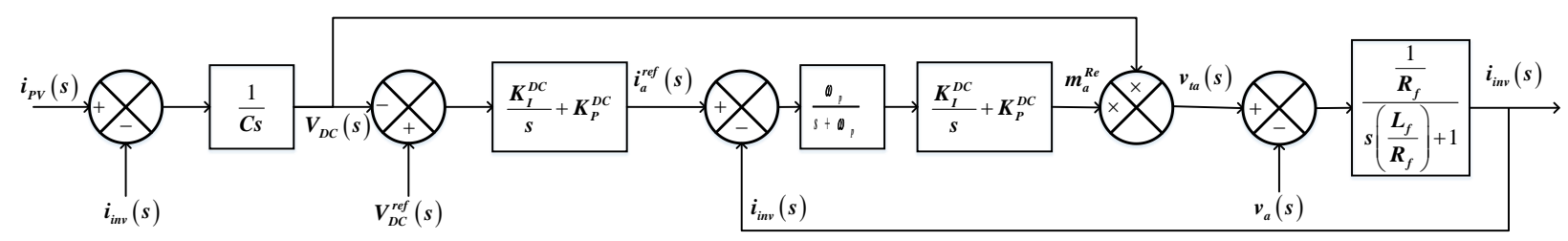

Figure 23. Control block diagram for active power loop

- To deal with unbalanced voltages all the duty cycles were calculated individually and was found to work for up to $3 \%$ imbalance in voltage magnitude.

- An iterative algorithm was used to find steady-state values of the PV-DER pertaining to a particular operating condition defined by PV module power output, DC link voltage, and grid voltage. 


\section{Sub-task 3.2.4:}

Investigate utility use-case with UAG input.

\section{Summary of activities:}

- The UAG recommended a frequency response use-case in which a transmission fault affects distributed solar PV inverters embedded in the distribution feeder. The objective of the study is to determine the degree to which an aggregated solar PV model, such as PVD1, captures the aggregated behavior of the PV inverters embedded in the distribution feeder.

- The concern is that high-penetration solar PV levels could lead to poor frequency response following a major transmission disturbance, which could initiate a cascading outage.

- Since the utility does not currently have the capability to study the combined T\&D system in detail, there is significant interest in validating an aggregated solar PV model, such as PVD1, under high-penetration scenarios.

- The utility transmission model (PSSE RAW and DYR data) and the utility feeder models were used to evaluate the use-case.

- Eight copies of the utility feeder were placed at the pre-determined transmission substation.

- The PV inverter locations and power outputs were determined by NREL based on their QSTS studies.

- The following simulations represent a high-penetration scenario with 237 single-phase PV inverters embedded in the distribution feeder. The distribution feeder has $3.1 \mathrm{MW}$ of load.

- The high-penetration (80\%) scenario causes reverse power flow of $1.1 \mathrm{MW}$ from the feeder up into the transmission system during peak solar irradiance, when the feeder load is below its peak.

- Under the high-penetration scenario, sustained post-fault oscillations were observed following a three-phase fault. Note that the PV inverters were assumed to be legacy inverters that would trip following a severe fault.

A three-phase low-impedance fault $(R=0.01 \mathrm{pu})$ was placed at the pre-determined highside transmission bus and cleared within 0.10 seconds ( 6 cycles)
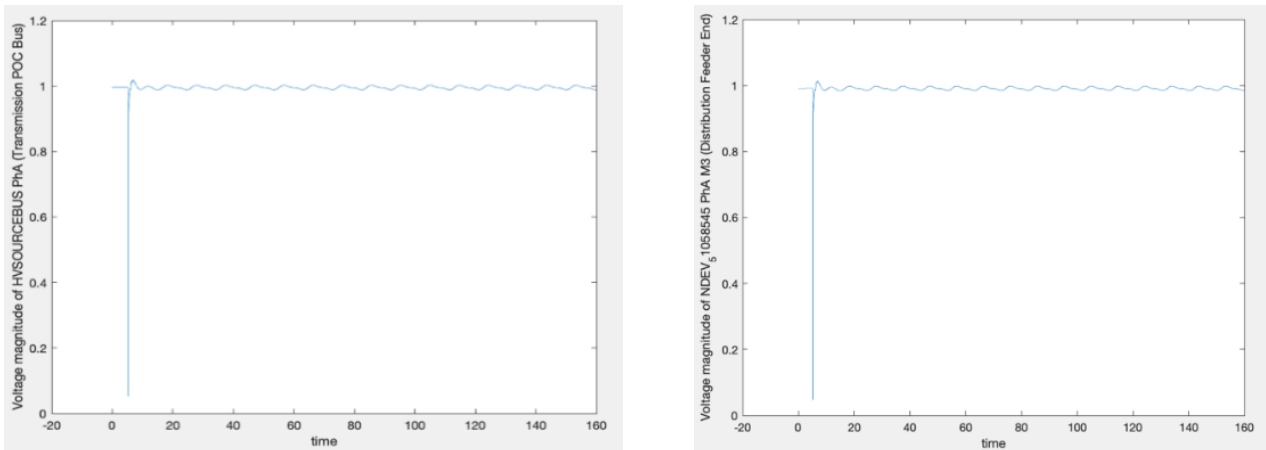

Figure 24. Left: Voltage magnitude (pu) vs. time at high-side transmission bus for three-phase transmission fault with 0.10 second duration. Right: Voltage magnitude (pu) vs. time at end of utility feeder for three-phase transmission fault with 0.10 second duration 

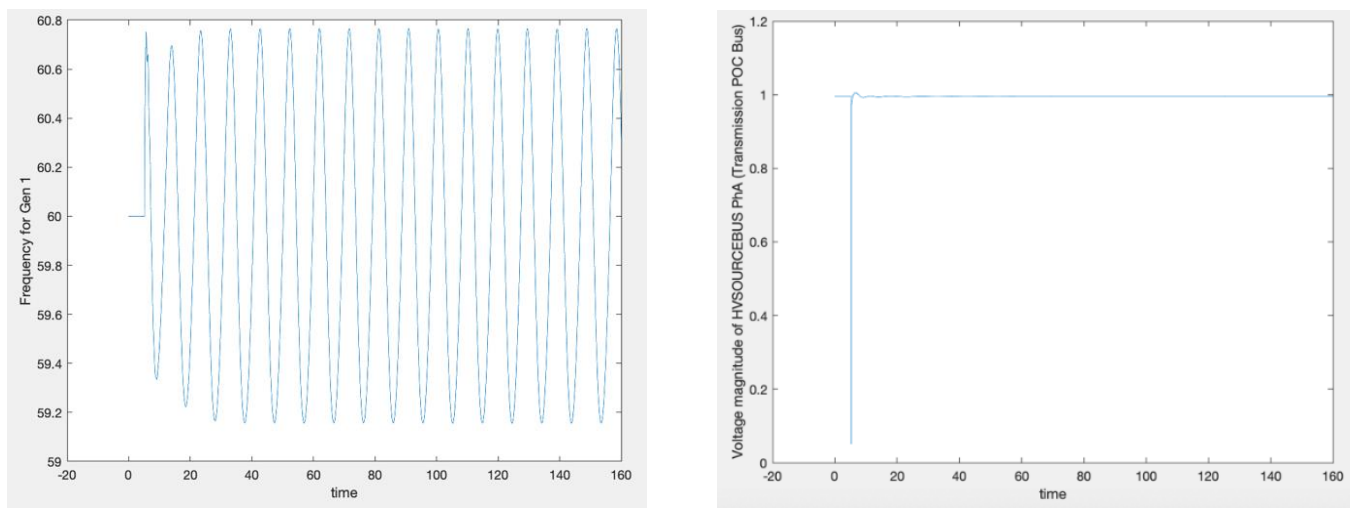

Figure 25. Left: Frequency $(\mathrm{Hz})$ vs. time at nearby bulk power generator bus for three-phase transmission fault with 0.10 second duration. Right: Voltage magnitude (pu) vs. time at high-side transmission bus for single-phase transmission fault with 0.10 second duration.

- Post-fault sustained oscillations were observed in the faulted transmission bus and distribution feeder node voltage magnitudes.

- Sustained frequency oscillations were observed in the transmission system under the high-penetration scenario due to tripping of the distributed PV inverters embedded in the distribution feeder.

A single-phase low-impedance fault $(R=0.01 \mathrm{pu})$ was placed at the pre-determined highside transmission bus on phase $A$ and cleared within 0.10 seconds $(6$ cycles)
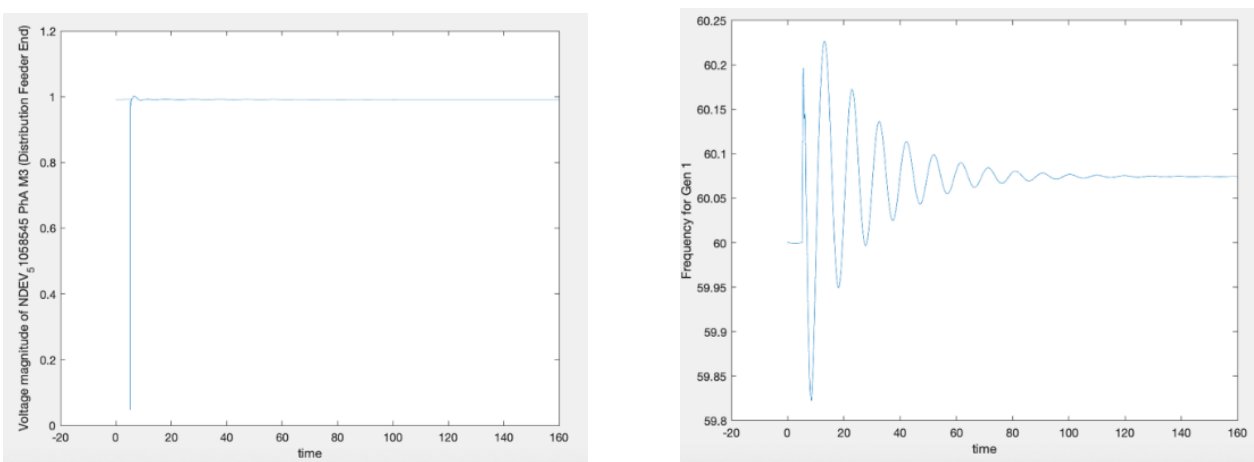

Figure 26. Left: Voltage magnitude (pu) vs. time at end of utility feeder for single-phase transmission fault with 0.10 second duration. Right: Frequency $(\mathrm{Hz})$ vs. time at nearby bulk power generator bus for singlephase transmission fault with 0.10 second duration.

- Post-fault damped oscillations were observed in the faulted transmission bus and distribution feeder node voltage magnitudes following the single-phase fault on phase A.

- Damped frequency oscillations were observed in the transmission system under the high-penetration scenario due to partial tripping of the distributed PV inverters embedded in the distribution system. 


\section{Comparison of Transmission-only model vs. combined T\&D model:}

- Without the detailed distribution feeder model, a transmission planning engineer can only model the aggregate behavior of distributed PV inverters with a lumped PV inverter model connected to the high-voltage substation bus.

- The following plots compare the T-only (blue curve) simulation vs. the combined T\&D (red curve) simulation.

- Both models show sustained oscillations following the severe three-phase transmission fault under the high-penetration (80\%) scenario with $1.1 \mathrm{MW}$ of reverse power flow.
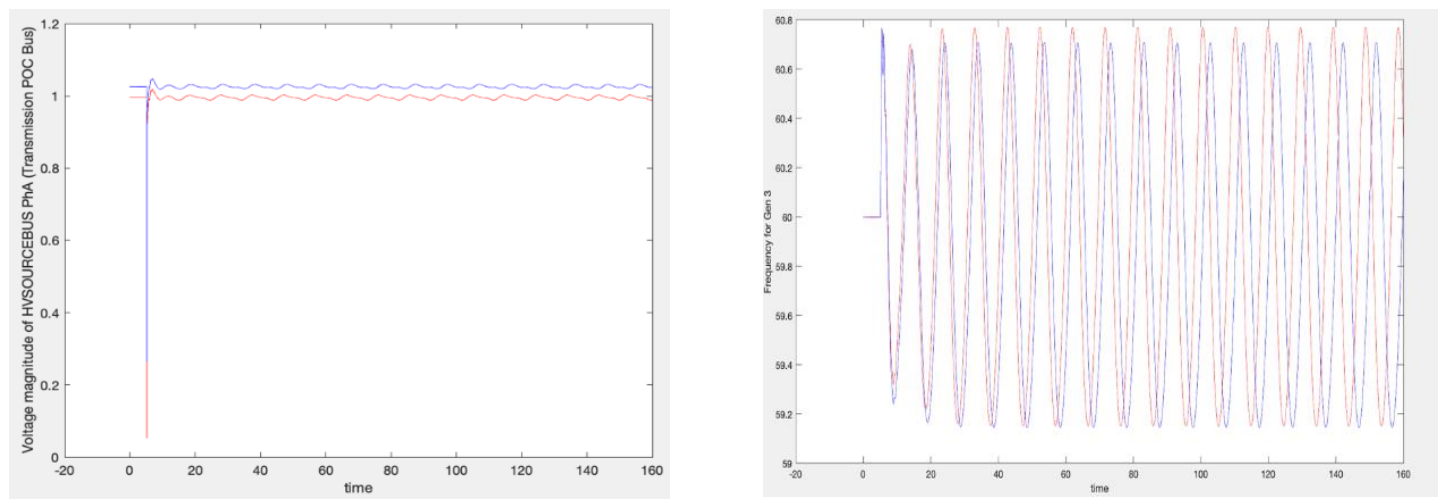

Figure 27. Left: Voltage magnitude (pu) vs. time at high-side transmission bus for three-phase transmission fault with 0.10 second duration (T-only in blue, combined T\&D in red). Right: Frequency $(\mathrm{Hz})$ vs. time at nearby bulk power generator bus for three-phase transmission fault with 0.10 second duration

(T-only in blue, combined T\&D in red).

- For this specific scenario, the T-only model shows a similar post-fault oscillation as the combined T\&D model. However, this result cannot be generalized to other operating conditions, nor other penetration scenarios. In other words, there is no guarantee that a T-only model can capture the dynamics of a combined T\&D system for all operating conditions.

- In general, unbalanced operating conditions cannot be captured by traditional positive-sequence transient stability simulators. Therefore, combined T\&D models that assume positive-sequence balanced operation in the transmission system will fail to represent the true dynamics of a realistic power system under unbalanced conditions.

\section{Sub-task 3.2.5:}

Assess protection system impacts based on use case scenarios

\section{Summary of activities:}

Developed a protection use-case that models transmission system network (T) and distribution system network (D) with high penetration of distributed generation based on rooftop photo-voltaic (PV) panels. The protection use-case was used to determine 
whether $T+D$ system and protection modeling is required for transmission network or distribution network protection studies, or the use of equivalents is sufficient.

\section{Objectives:}

To investigate whether, in power systems with high penetration of distributed generation based on rooftop photo-voltaic (PV) panels, the following assumptions are true:

- That any fault contingency in the transmission network is completely addressed and cleared by the transmission network protection and no coordination with the distribution network protection is necessary.

- That any fault contingency in the distribution network is completely addressed and cleared by the distribution network protection and no coordination with the transmission network protection is necessary.

If the above assumptions are true for a number of different observations, then the following assertions are correct:

- Complete and correct analysis of transmission network protection of systems with high penetration of distributed generation based on rooftop photo-voltaic (PV) panels is achievable exclusively by the analysis of transmission system models.

- Complete and correct analysis of distribution network protection of systems with high penetration of distributed generation based on rooftop photo-voltaic (PV) panels is achievable exclusively by the analysis of distribution system models.

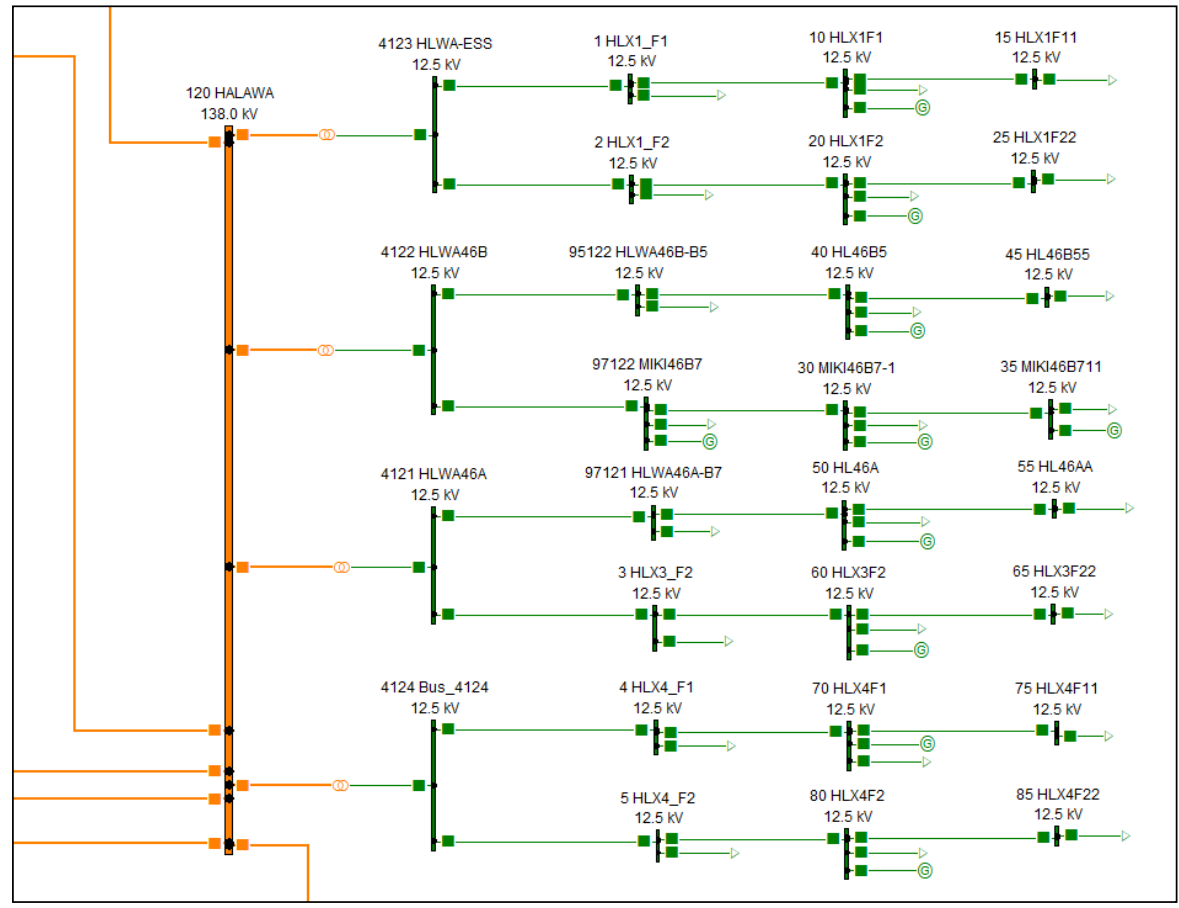

Figure 28. Made-up 138 kV Substation 120 Halawa in O'ahu HECO System with Added Fake Distribution System 
The tested distribution fault scenarios are the following:

1. Close-in three-phase fault with fault resistance of $1 \mathrm{ohm}$ in distribution feeder 4122 97122, with primary protection disabled, high summer.

2. Close-in three-phase fault with fault resistance of $1 \mathrm{ohm}$ in distribution feeder 4122 97122, with primary protection enabled, high summer.

3. Close-in three-phase fault with fault resistance of $1 \mathrm{ohm}$ in distribution feeder 4122 97122, with primary protection disabled, light spring.

4. Close-in three-phase fault with fault resistance of $1 \mathrm{ohm}$ in distribution feeder 412297122, with primary protection enabled, light spring.

Here it is a token of results of distribution fault scenario 1 focusing in distance protection
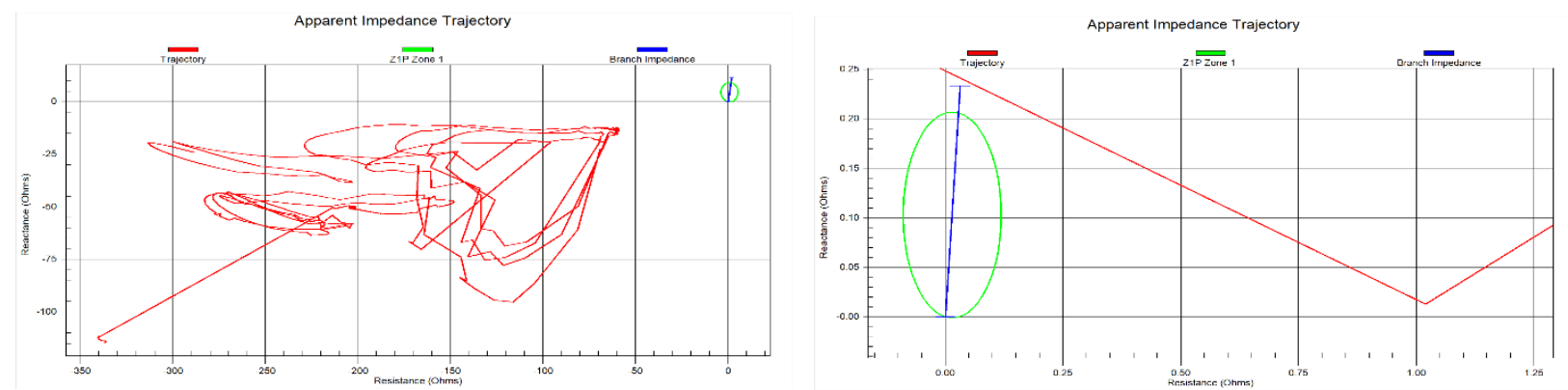

Figure 29. Left: Apparent impedance trajectory of distance protection zone 1 in transmission line 120-130. Apparent impedance trajectory fell into the load zone of the protection. Right: Detail of the apparent impedance trajectory of distance protection zone 1 in distribution feeder 4122-97122. The result is similar in tests 1 and 3 , only differing that in the test 3 the feeder distance protection identified the fault and cleared it.

The above two graphs show that the fault in the distribution feeder does not affect the distance protection in the transmission side. The rest of the protection studies 1,2, 3 and 4 corroborate this observation.

The tested transmission fault scenarios are the following:

5. Close-in three-phase fault with fault resistance of $1 \mathrm{ohm}$ in transmission line 120 Halawa to a remote adjacent substation, with primary protection disabled, high summer.

6. Close-in three-phase fault with fault resistance of $1 \mathrm{ohm}$ in transmission line 120 Halawa to a remote adjacent substation, with primary protection enabled, high summer. 

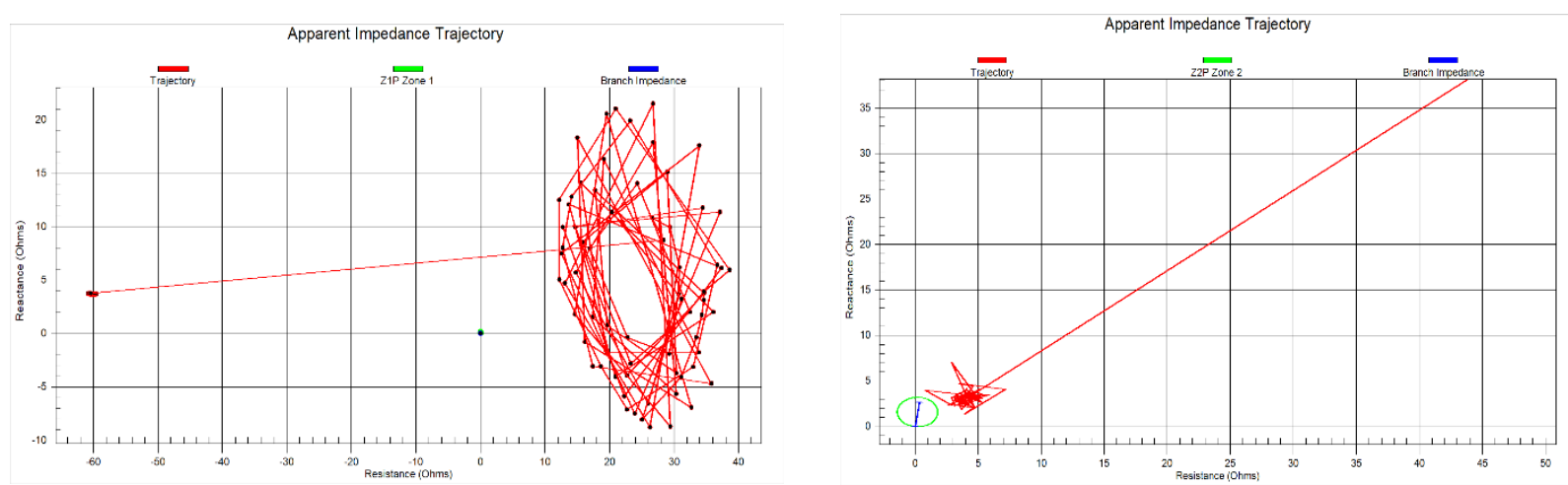

Figure 30. Left: Apparent impedance trajectory of distance protection zone 2 in feeder 4122-97122. Distance zone 2 characteristic sat at the origin of the R-X graph. Apparent impedance trajectory fell into the load zone of the protection. Right: Apparent impedance trajectory of distance protection zone 2 in an adjacent remote transmission line. Distance zone 2 identifies the fault in the remote line and opens its local breaker.

The above two graphs show that a fault in the transmission line does not affect the operation of distance protection in the distribution side. In addition, the distance transmission protection shows that coordination (zone 2 identifies a close-in fault in a remote line) is not affected greatly by the in-feed contribution to the fault by the distribution network. Results of case 5 corroborate this observation.

\section{Conclusions:}

- Any fault contingency in the transmission network is completely addressed and cleared by the transmission network protection and no coordination with the distribution network protection is necessary. Therefore, complete and correct analysis of transmission network protection of systems with high penetration of distributed generation based on rooftop photo-voltaic (PV) panels is achievable exclusively by the analysis of transmission system models.

- Any fault contingency in the distribution network is completely addressed and cleared by the distribution network protection and no coordination with the transmission network protection is necessary. Therefore, complete and correct analysis of distribution network protection of systems with high penetration of distributed generation based on rooftop photo-voltaic (PV) panels is achievable exclusively by the analysis of distribution system models.

\section{Caveat:}

The PV model in PSS®E has serious numerical stability issues. Siemens PTI has adviced that their models present voltage angle spikes due to the nature of the solving algorithm. There, voltage is not a state variable, but an algebraic variable. This means that the voltage angle is subject to quick change, which affect the computation of current, power and frequency. Similarly, the amount of test cases tested could be increased to further corroborate this assumption. 


\section{Milestone 3.2:}

Largest average magnitude percentage voltage error for any time step (except first two cycles after switching event) should meet the success value of less than $1 \%$ error in voltage magnitude for at least 4 out of 5 scenarios.

Voltage magnitude error $=\mid$ RMS value of EMT waveform - magnitude of TS phasor $\mid$

Summary of activities:

- The EMT simulations were conducted in PSCAD.

- Due to the EMT modeling and simulation constraints, a small test system was built in PSCAD for the milestone evaluation.

- The IEEE 13 Node Test Feeder was connected to bus 8 of the nine-bus transmission system.

- Bolted three-phase and single-phase to ground faults were simulated in PSCAD and TS3ph.

- The PSCAD simulation used the detailed PV inverter generator model (REGCA/PVGU1) and electrical controller (REECB1) based on NREL's earlier PSCAD project: User Guide for PV Dynamic Model Simulation Written on PSCAD Platform by E. Muljadi, M. Singh, and V. Gevorgian.

- Some modifications of the single-phase PSCAD model were required before the model could reliably regulate it's real power output.

- The TS3ph simulation used a detailed PV inverter model (REGC_A/PVGU1D), which has been validated against the REGC_A inverter model in PowerWorld.

- Two PV locations were used for scaling the total PV output: node 650 (low-side bus of substation) and node 652 (near the end of the feeder).

- Three PV penetration scenarios were investigated.

- The net feeder load at the substation is shown in the table below for the original feeder model without PV generation and the three levels of PV penetration.

\begin{tabular}{|l|r|r|r|r|r|r|}
\hline & phA (kW) & $\begin{array}{r}\text { phB } \\
(\mathbf{k W})\end{array}$ & $\begin{array}{r}\text { phC } \\
\mathbf{( k W )}\end{array}$ & $\begin{array}{r}\text { phA } \\
\mathbf{( k V a r )}\end{array}$ & $\begin{array}{r}\text { phB } \\
(\mathbf{k V a r})\end{array}$ & $\begin{array}{r}\text { phC } \\
(\mathbf{k V a r})\end{array}$ \\
\hline Zero-PV & 1034.16, & 1233.87 & 1265.56 & 604.94 & 453.28 & 702.18 \\
\hline Low-PV & 669.35 & 986.70 & 877.74 & 500.59 & 443.24 & 747.14 \\
\hline Med-PV & 316.13 & 735.15 & 506.52 & 419.43 & 441.24 & 793.85 \\
\hline High-PV & -397.89 & 249.71 & -192.13 & 300.47 & 435.60 & 930.19 \\
\hline
\end{tabular}

- Note that the detailed bulk power generator models for transient stability ignore stator transients, while the electromagnetic transient generator models include stator transients. This well-understood difference prevents a straightforward comparison between TS and EMT simulations.

- The following figure shows the difference between the two simulation environments, as well as a comparison within the TS environment between TS3ph and PSSE. In 
this case, no PV is connected to the system, so the comparison highlights the difference between the synchronous generator modeling assumptions.

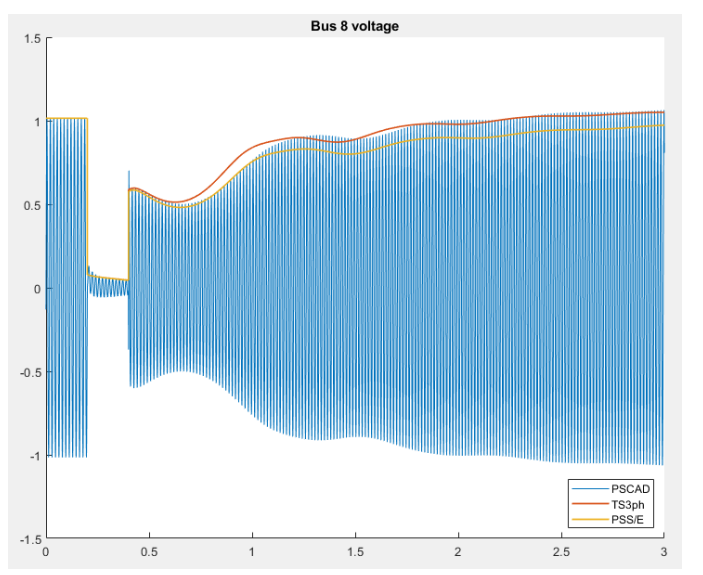

Figure 31. Voltage at Bus 8: PSSE (yellow), TS3ph (red), PSCAD (blue) comparison for three-phase fault

- The difference in dynamic behavior due to the inconsistent assumptions for stator transients prevents the use of detailed generator models when comparing PV inverter models implemented in TS vs PV inverter models implemented in EMT.

- To isolate the PV inverter model impacts on the dynamic behavior of the combined T\&D test system, the team was forced to use a classical (reduced-order) generator model with a very large inertia constant. Under these conditions, the bulk power generator model no longer causes the differences shown in the above figure when comparing TS and EMT simulation results.

- The following comparison cases demonstrate the achievement of Milestone 3.2, which had a target of a $1 \%$ maximum voltage difference between the TS voltage magnitude and the envelope of the EMT voltage.

Case 1: Low-PV penetration: node 650: $750 \mathrm{~kW}$, node $652: 250 \mathrm{~kW}$
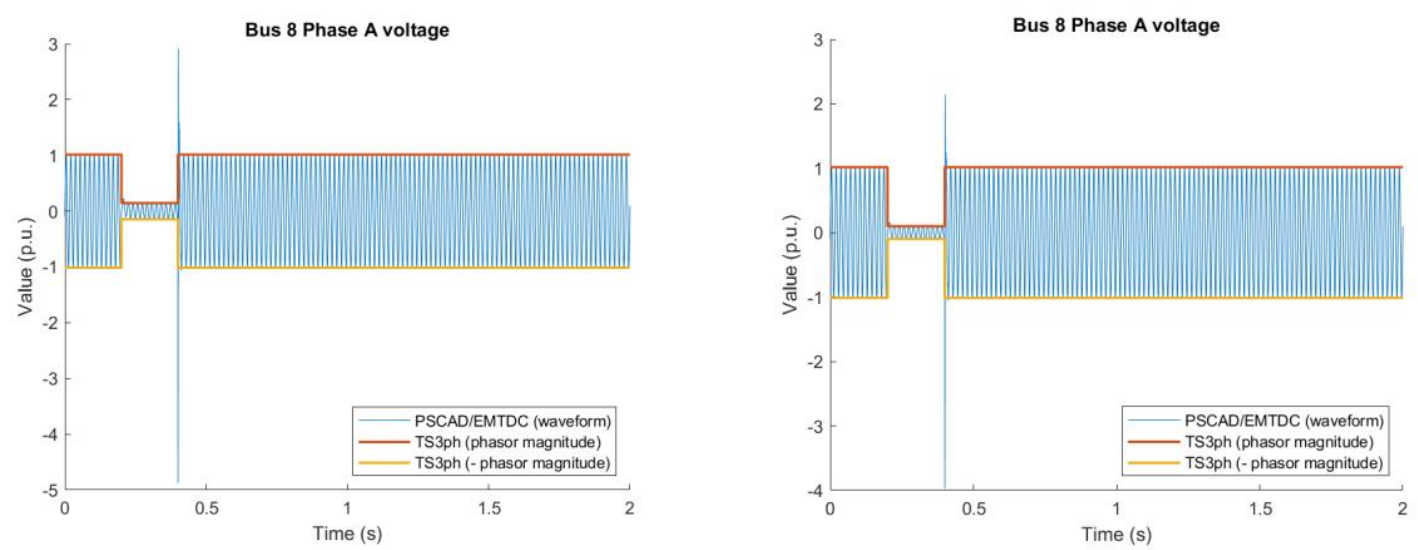

Figure 32. TS3ph voltage phasor magnitude compared to PSCAD waveform for low penetration. Left: Comparison for Three-phase fault at high-side bus of substation. Right: Comparison for Phase A to ground fault at high-side bus of substation. 
Case 2: Medium-PV penetration: node 650: $1500 \mathrm{~kW}$, node 652: $500 \mathrm{~kW}$
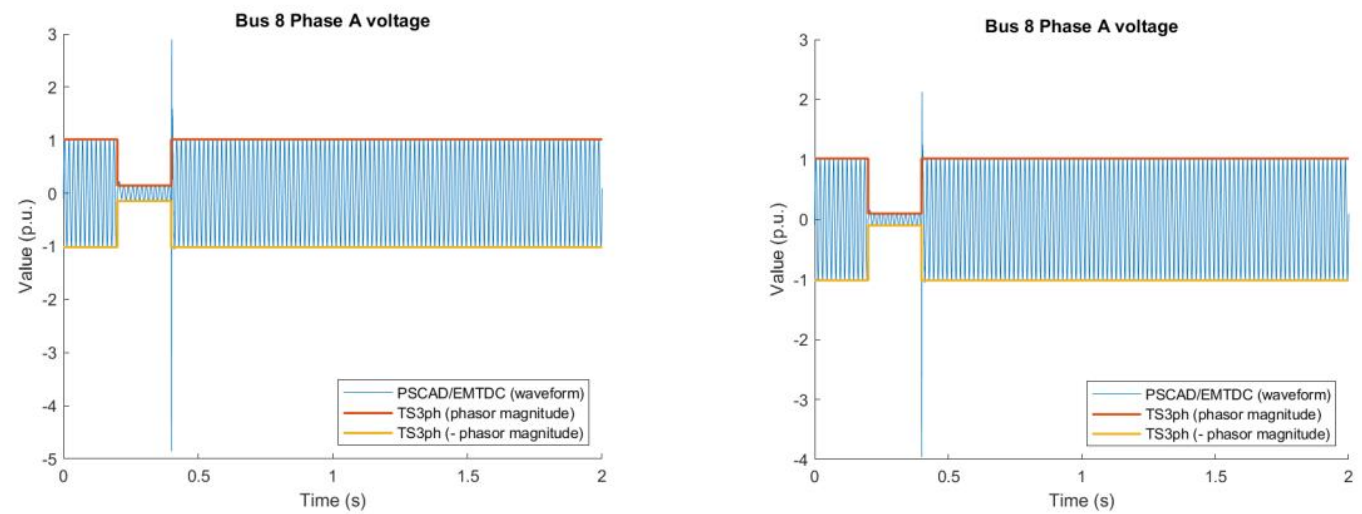

Figure 33. TS3ph voltage phasor magnitude compared to PSCAD waveform for medium penetration. Left: Comparison for Three-phase fault at high-side bus of substation. Right: Comparison for Phase A to ground fault at high-side bus of substation.

Case 3: High-PV penetration: .node 650: $3000 \mathrm{~kW}$, node 652: $1000 \mathrm{~kW}$
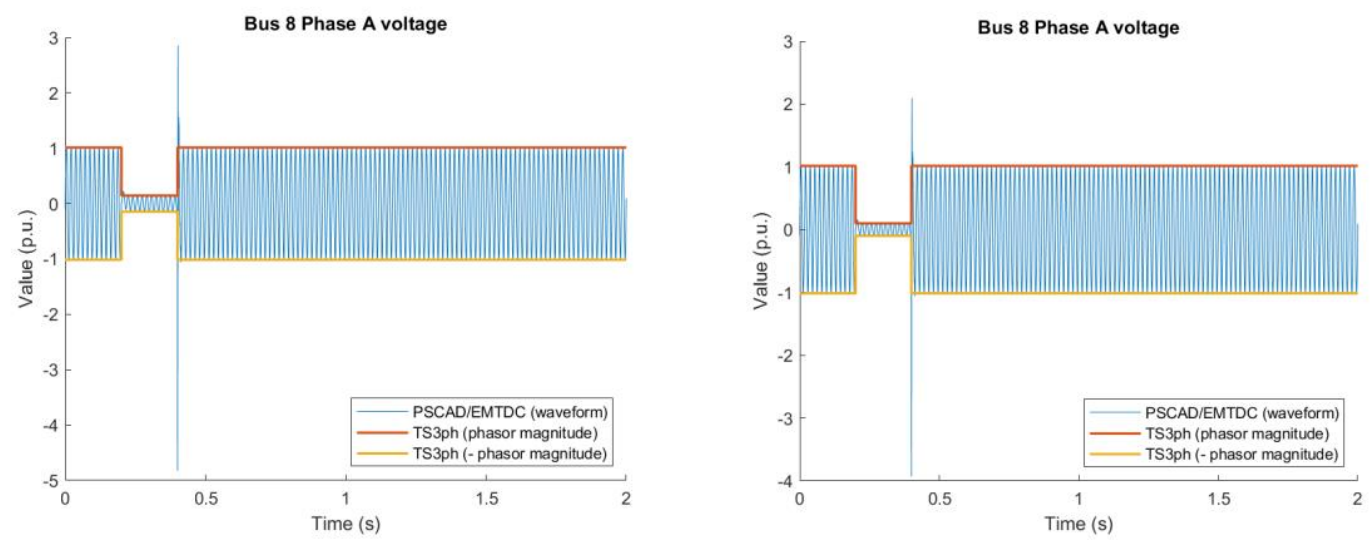

Figure 34. TS3ph voltage phasor magnitude compared to PSCAD waveform for high penetration. Left: Comparison for Three-phase fault at high-side bus of substation. Right: Comparison for Phase A to ground fault at high-side bus of substation.

Milestone 3.2: This milestone is fully complete. The TS detailed PV inverter model simulation results matched the EMT detailed PV inverter model simulation results within the $1 \%$ maximum voltage difference limit for both three-phase and singlephase faults under all three penetration levels, which yielded 6 unique scenarios.

\section{Task 3.3:}

Analyze utility use case with distribution system state estimator (DSSE).

\section{Sub-task 3.3.1:}

Verify steady-state behavior of DSSE on other test feeders against OpenDSS 


\section{Summary of activities:}

- The DSSE simulations were conducted with two different solution algorithms: SemiDefinite Programming (SDP) and Weighted Least Squares (WLS).

- The test case was a modified version of the IEEE 8500 Node Test Feeder in which secondary circuits (customer service drops) and customer transformers were eliminated. Loads were aggregated for each customer transformer and located on the primary distribution feeder in place of the customer transformer. The bus count for the modified system was 2475 buses, which included 3708 single-phase nodes.

- The test feeder power flow was solved via OpenDSS and following noise values were added to the voltages and currents to mimic noisy measurements with significant measurement latency. i) Voltage magnitude measurement noise: $2 \%$, ii) Current magnitude measurement noise: 10\%, iii) Phasor Measurement Unit (PMU) noise: $0.2 \%$.

- A PMU was assumed to be located in the substation. All other measurements were assumed to be collected from smart meters.

\section{Sub-task 3.3.2:}

Validate behavior of DSSE during dynamic disturbances on IEEE Test Feeders against TS3ph.

\section{Summary of activities:}

- The test was carried out on the modified IEEE 8500 node distribution test feeder with 2475 buses (3708 single-phase nodes).

- TS3ph was used to simulate a disturbance in the transmission network, which lowered the substation voltage. The goal was to investigate the ability of DSSE to estimate the new state of the system following the disturbance before the new smart meter interval data is received. This test assumed the smart meter interval measurement data came from the network conditions related to the pre-disturbance system, which had a higher substation voltage, hence higher voltages throughout the feeder. In this test, only the PMU measurement data came from the network conditions related to the postdisturbance system.

- Goal: Evaluate the DSSE errors immediately following a disturbance to see if the updated PMU measurements are sufficient to estimate the distribution node voltages even without updated smart meter measurements.

- The pre-disturbance substation voltage was $1.05 \mathrm{pu}$. The post-disturbance substation voltage was $0.95 \mathrm{pu}$.

- Both SDP and WLS fail to meet the $1 \%$ voltage magnitude error target.

\section{Sub-task 3.3.3:}

Validate behavior of DSSE during dynamic disturbances on utility test feeders against utility disturbance records. 


\section{Modification to PMP:}

Utility disturbance data was not available. Hence, this task was removed from PMP at the start of BP3.

\section{Sub-task 3.3.4:}

Investigate utility use-case with DSSE based on Utility Advisory Group input

Summary of activities:

- The test was carried out on the modified IEEE 8500 node distribution test feeder with 2475 buses (3708 single-phase nodes).

- Three penetration scenarios, relative to peak feeder load, were simulated: $30 \%, 50 \%$, $75 \%$. The feeder load for the testing snapshot was assumed to be $60 \%$ of the peak feeder load due to the timing of peak solar irradiance.

- Peak feeder load, without solar PV generation, is as follows

○ A: 2905.4 -j224 KVA, B: 2945.8-j184.6 KVA, C: 3664.0 +j63.7 KVA

- For PV penetration of $75 \%$ relative to peak feeder load, when the load is only $60 \%$ of the peak (during the day, when the solar irradiance is at it's peak), there is a reversal in the power flow at the substation. The consumption of real power by the distribution feeder becomes negative as shown below.

○ A: -366.4-j992.9 KVA, B: -371.34-j970.51 KVA, C: -461.907-j872.7 KVA

\section{Test cases:}

- Case 1: PV penetration is $30 \%$ of peak feeder load

- Case 2: PV penetration is $50 \%$ of peak feeder load

- Case 3: PV penetration is $75 \%$ of peak feeder load

- The following plots show voltage magnitude and voltage angle errors for SDP (red asterisk) and WLS (blue asterisk)

- Both SDP and WLS meet the $1 \%$ voltage magnitude error target for all three cases. 

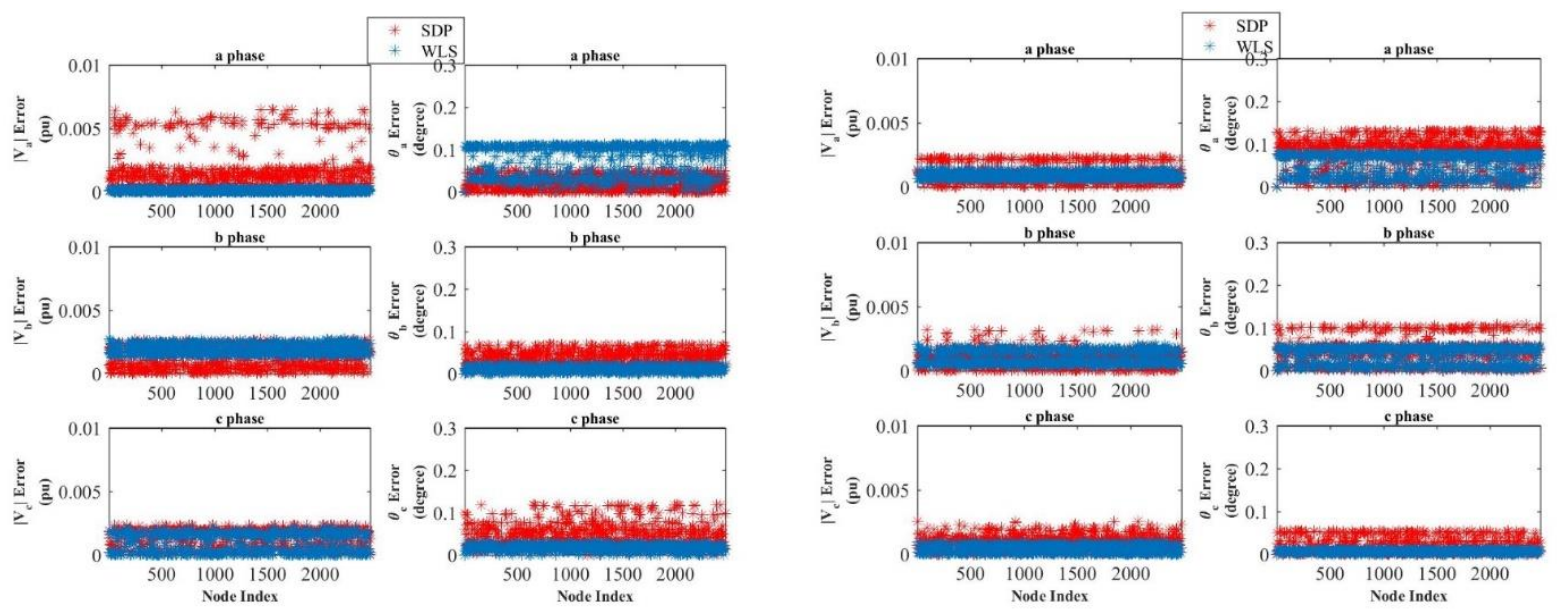

Figure 35. Left: Case 1 Voltage magnitude and angle errors for PV penetration of 30\%. Right: Case 2 Voltage magnitude and angle errors for PV penetration of $50 \%$.

\section{Milestone 3.3:}

- At least 2 out of 3 simulations of PV penetration scenarios should meet the success rate of less than $1 \%$ voltage magnitude error.

\section{This milestone is fully complete.}

\section{Significant Accomplishments and Conclusions}

\section{Tool-Suite Development}

The goal of the project is to address the following challenges/barriers,

$\checkmark$ The needs to improve understanding of transmission-distribution interactions
$\checkmark$ Create an awareness of real-time distribution system operating conditions
$\checkmark$ To capture time series dynamics across multiple scales within associated tools

To this end, the team developed a suite of software tools that imparts a holistic understanding of the steady-state and transient behavior of transmission-distribution interaction under high PV penetration levels along with real-time monitoring of the distribution system and integration of system protection.

Existing steady-state models fails to capture the important spatial variations for PV (and load) and the potential for heterogeneous inverter types and set points across a distribution feeder. In particular, during voltage disturbances, inverters connected at different points on a feeder can see very different voltage profiles, suggesting a need for either location-based voltage ride-through settings or wider ride-through envelopes for distribution connected systems than those used at the bulk power level. The developed 
T\&D steady-state tool uses a far more detailed representation of the distribution system, including multi-branched network representation to capture spatial differences and enable heterogeneity in the PV inverter fleet. This work is novel in that it is believed to be the first integrated distribution and transmission system modeling effort utilizing HPC that can accurately describe the transmission level impacts of large amounts of distributed energy resources such as PV.

The importance and need for simultaneous transmission-distribution (T-D) dynamics and protection simulation tool stems from the fact that traditional transmission dynamics simulators crudely coupled to distribution dynamics simulators will not produce faithful representations of dynamic grid behavior once distribution networks become more dynamic. Time-series analysis, power flow snapshots, and traditional fault studies fail to capture the necessary dynamics for identifying stability limits and protection limits for planning studies, especially under a high-penetration PV scenario. The team developed a new solution technique that solves the transmission-distribution system equations simultaneously (superior simultaneous "single equation" solution protocol) as opposed to merely connecting different transmission dynamics simulators with distribution dynamics simulators that exchange information at the end of every step (inferior serial or parallel interaction protocols).

Distribution system state estimator (DSSE) aims to minimize the weighted least squares problem that is typically nonlinear and non-convex. The Gauss-Newton (G-N) method is often applied to solve such a problem. However, the solution, if found, is always a local optimum. In some cases, the iteration process cannot even converge. Hence, a semidefinite programming (SDP) formulation is developed in this work to solve the DSSE problem, which is relaxed to a solvable convex problem by the semidefinite relaxation (SDR). A major benefit of the developed DSSE is its robustness, which will be helpful in the future of dynamic distribution operations with high penetration PV.

\section{Use Case Development}

In addition to tool-suite development, the team engaged with UAG members to better understand the challenges power industry is currently facing. The team then identified and developed use cases. HECO provided data for both transmission and the distribution system. This dataset allowed the team to,

- Test the developed tool-suite's robustness

- Provide valuable feedback to HECO

- Understand what type of analysis requires T\&D co-simulation

The following figure illustrates the diversity of the developed tools and the type of analysis that can be performed - illustrated through use cases developed in this project. 


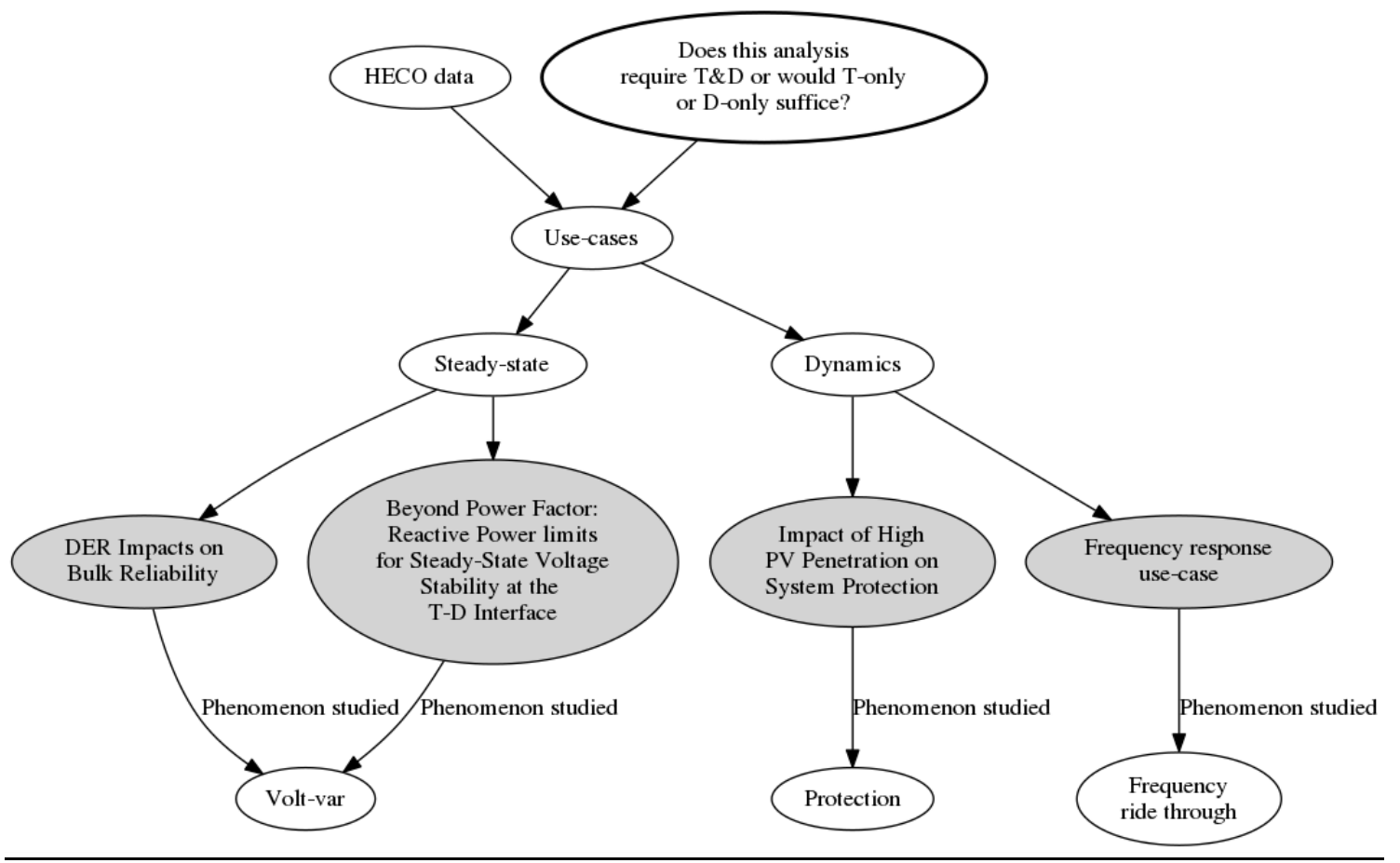

Figure 36. List of use-cases, phenomenon studied by each use-case with the goal of addressing the question "does this analysis require T\&D or would T-only or D-only analysis suffice?"

\section{Inventions, Patents, Publications, and Other Results}

\section{Final Deliverables:}

FD-1: A final report on the methods, technologies, and findings of this project.

FD-2: A website to disseminate information on the scope, mission, and the achieved milestones.

$\checkmark$ Website: https://www.anl.gov/es/sunlamp-anl

FD-3: A minimum of six publications submitted to peer review journals/conference proceedings.

This deliverable is complete. The published papers are given below.

1. B. Palmintier, E. Hale, B. M. Hodge, K. Baker, and T. Hansen, "Experiences integrating transmission and distribution simulations for DERs with the Integrated Grid Modeling System (IGMS)", in Proceedings of the $19^{\text {th }}$ Power Systems Computation Conference (PSCC' 16) Genoa, Italy, 2016.

2. K. Balasubramaniam, S. Abhyankar, "A combined transmission and distribution system co-simulation framework for assessing the impact of volt/var control on transmission system," IEEE Power \& Energy Society General Meeting, 2017, pp. 1-5, IEEE. 
3. H. Jain, B. Palmintier, I. Krad, and D. Krishnamurthy, "Studying the Impact of Distributed Solar PV on Power Systems using Integrated Transmission and Distribution Models," IEEE PES T\&D Conference and Exposition, Denver, CO, 2018.

4. B. Palmintier, I. Krad, and D. Krishnamurthy, "Impacts of Distributed Solar Advanced Inverters on Transmission Voltage and Reactive Power," in Proc. 8th International Workshop on the Integration of Solar Power into Power Systems, Stockholm, Sweden, 2018.

5. S. Abhyankar, K. Balasubramaniam, and B. Cui, "Load Model Parameter Estimation by Transmission-Distribution Co-Simulation," Power Systems Computation Conference (PSCC), June 2018.

6. S. Plathottam, S. Abhyankar, and P. Hazra, "Dynamic Modeling of Solar PV Systems for Distribution System Stability Analysis," Innovative Smart Grid Technologies (ISGT), Washington, DC, February 2019.

7. S. Lei and A. J. Flueck, "Accelerating Electromagnetic Transient Simulation for Power System Stability Studies Using Frequency Shifted Companion Signal and Frequency Response Optimized Integrators," IEEE Power \& Energy Society General Meeting, 2019.

$\checkmark$ FD-4: Public release of steady-state transmission-distribution analysis tool that includes NREL's IGMS tool and ANL's PFLOW tool.

$\checkmark$ PFLOW: https://github.com/pflow-team/PFLOW

$\checkmark$ FESTIV: https://github.com/NREL/FESTIV_MODEL

$\checkmark$ IGMS: https://github.com/NREL/IGMS (NREL Tech Transfer currently engaged on open-sourcing the IGMS platform. Awaiting final approvals to make the IGMS GitHub repo public.

\section{$\checkmark \quad$ All four of the final deliverables are fully complete.}

\section{Path Forward}

As power industry continues to evolve, so should the analysis tools. The team hopes that the research community can benefit from and extend the progress that was made over the course of this project. To this end, the publications and the open sourced software that resulted from the project will provide a solid platform upon which future work can be built. The project website will act as a single point for all the relevant information such as links to publications, GitHub repositories for open sourced software, etc. The team also hopes that the engagement with HECO and PG\&E over the course of this project through UAG will act as a catalyst in industry adoption of T\&D co-simulation analysis. 


\section{References}

And2013 K. Anderson, J. Du, A. Narayan, and A. El Gamal, "GridSpice: A distributed simulation platform for the smart grid," in 2013 Workshop on Modeling and Simulation of Cyber-Physical Energy Systems (MSCPES), 2013, pp. 1-5.

Dai2014 J. Daily, J. Fuller, S. Ciraci, A. Fisher, L. Marinovic, and K. Agarwal, "FNCS: Framework for Network Co-Simulation," Richland, WA, January 22, 2014.

Kos2010a D. N. Kosterev, A. Meklin, J. Undrill, B. Lesieutre, W. Price, D. Chassin, R. Bravo, and S. Yang, "Load modeling in power system studies: WECC progress update," in 2008 IEEE Power and Energy Society General Meeting - Conversion and Delivery of Electrical Energy in the 21st Century, 2008, pp. 1-8.

Kos2010b D. Kosterev, "Load Modeling in WECC," presented at the SRWG Modeling Workshop, Salt Lake City, UT, November 2010.

Sch2011 D. A. Schoenwald, et al., "Final report for 'High performance computing for advanced national electric power grid modeling and integration of solar generation resources,' LDRD Project No. 149016," Sandia National Laboratories, Albuquerque, NM, SAND2011-0890, February 2011.

Ell2011 A. Ellis, M. Behnke, and C. Barker, "PV System Modeling for Grid Planning Studies," Sandia National Laboratories, Albuquerque, NM, 2011.

EPSR EPRI Smart Grid Resource Center Use Case Repository, available at http://smartgrid.epri.com/Repository/Repository.aspx

ISGAN2014 International Smart Grid Action Network, TSO-DSO interaction:

An Overview of current interaction between transmission and distribution system operators and an assessment of their cooperation in smart grids, September 2014.

PJM101 "PJM - LMP/FTR 101." <http://www.pjm.com/ /media/training/corecurriculum/ip-Imp-101/lmp-training.ashx>.

Hudson2012 Raymond Hudson and Gerd Heilscher, PV Grid Integration - System Management Issues and Utility Concerns, Proceedings of the PV Asia Conference, 2011.

Margo2014 Robert Margolis, The long-term vision for solar in United States, $6^{\text {th }}$ World Conference on Photovoltaic Energy Conversion, 2014.

Berdner2014 John Berdner, The impact of Smart Inverters: How Rule and Regulation will transform DG into Smart Systems. 


\section{Argonne}

\section{Energy Systems Division}

Argonne National Laboratory

9700 South Cass Avenue, Bldg. 362

Argonne, IL 60439

www.anl.gov 